\title{
Hydrodynamical description of first-order phase transitions: analytical treatment and numerical modeling
}

\author{
V.V. Skokov ${ }^{\mathrm{a}, \mathrm{b}, \mathrm{c}}$ and D.N. Voskresensky ${ }^{\mathrm{a}, \mathrm{d}}$

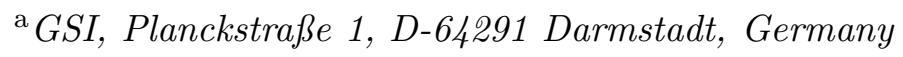 \\ ${ }^{\mathrm{b}}$ Frankfurt Institute for Advanced Studies, Universität Frankfurt, D-60438 \\ Frankfurt am Main, Germany \\ ${ }^{\mathrm{c}}$ Joint Institute for Nuclear Research, 141980 Dubna, Moscow Region, Russia \\ ${ }^{\mathrm{d}}$ Moscow Engineering Physical Institute, \\ Kashirskoe Avenue 31, RU-115409 Moscow, Russia
}

\begin{abstract}
Solutions of hydrodynamical equations are presented for an equation of state allowing for a first-order phase transition. The numerical analysis is supplemented by analytical treatment provided the system is close to the critical point. The processes of growth and dissolution of seeds of various sizes and shapes in meta-stable phases (like super-cooled vapor and super-heated liquid) are studied, as well as the dynamics of unstable modes in the spinodal region. We show that initially nonspherical seeds acquire spherical shape with passage of time. Applications to the description of the first-order phase transitions in nuclear systems, such as the nuclear gas-liquid transition occurring in low energy heavy-ion collisions and the hadron-quark transition in the high energy heavy-ion collisions are discussed. In both cases we point out the important role played by effects of viscosity and surface tension. It is shown that fluctuations dissolve and grow as if the fluid were effectively very viscous. Even in the spinodal region seeds may grow slowly due to viscosity and critical slowing down. This prevents the enhancement of fluctuations in the near-critical region, which is frequently considered as a signal of the critical point in heavy-ion collisions.
\end{abstract}

\section{Introduction}

The description of first-order phase transitions is usually based on phenomenological approaches. One constructs a thermodynamical potential depending on an order parameter, similar to that in the Landau theory of phase transitions. 
Exploiting the fact that the time evolution of the collective mode is usually slow compared to the dynamics of microscopic processes one introduces a time-dependent equation for the order parameter [1]

$$
\partial_{t} \phi=-\Gamma(\Delta)(\delta F / \delta \phi), \quad \Delta=\partial_{x_{1}}^{2}+\ldots+\partial_{x_{d}}^{2} .
$$

Here $d$ is the dimensionality of the space, $t$ is the time, $F$ is a thermodynamic functional expressed in proper variables (e.g., the $\phi$ - dependent correction to the Helmholtz free energy in $T, V$ variables), $T$ is the temperature, $V$ is the volume and $\phi$ is an order parameter. For a non-conserving order parameter $\Gamma(\Delta)=a_{0}$ and for a conserving one $\Gamma(\Delta)=a_{1} \Delta$. Appropriate quantities should be chosen as order parameters for different physical systems under consideration. These could be a macroscopic wave function for metallic superconductors and non-relativistic superfluids, as superfluid $\mathrm{He}^{4}$ and $\mathrm{He}^{3}$, dipole moment for ferroelectrics, magnetic moment for ferromagnetics, $\sigma, \omega, \rho, \pi$, $K$-mean fields for various phenomena of nuclear physics, etc.

There are many phenomena, where first-order phase transitions occur between phases with different densities. The description of such phenomena should be similar to that for a gas-liquid phase transition. Therefore it is worthwhile to find the solutions of corresponding hydrodynamical equations. Although some simplified analytical [2-4] and fragmentary two-dimensional numerical [5] solutions have been found, many problems still remain unsolved. In the general case one should construct an appropriate numerical scheme, which could describe the phenomenon. The hydrodynamical approach is fairly efficient for the description of heavy-ion collisions in a broad range of collision energy from SIS to RHIC energies (see e.g. [6-16]). As a simplification, most of three-dimensional hydrodynamical models use ideal hydrodynamics. Effects of viscosity and thermal conductivity are simulated then with the help of an artificially introduced friction between different components of the liquid. See, e.g. Ref. [7] for a three-fluid hydrodynamical scheme applicable in a broad energy range from SIS to SPS.

There are arguments [2-4] that the dynamics of a first-order phase transition is controlled by non-zero values of the kinetic coefficients. For nuclear systems transport coefficients are poorly known. Transport coefficients in nuclear matter have been evaluated in $[17,18]$ for temperatures and nucleon densities relevant for the nuclear gas-liquid (NGL) phase transition. Estimates [17] have demonstrated that the bulk viscosity is much smaller than the shear viscosity. Equations of non-relativistic non-ideal hydrodynamics have been solved to construct a description of heavy-ion collisions at SIS energies [6]. Some models, $[11,12]$, describing the expansion of matter at RHIC energies solve equations of relativistic non-ideal hydrodynamics in two spatial dimensions and indicate that effects of viscosity in the state of strongly coupled quark-gluon plasma (sQGP) are minor. One concluded that the ratio of the shear viscosity to the 
entropy density is $\eta / s<0.2$, see [12]. For certain materials, such as helium, nitrogen and water the experimental values for this ratio $\eta / s$ show a minimum at the phase transition [19]. The properties of the bulk viscosity near the hadronic-to-sQGP phase transition critical point (endpoint) are discussed in Refs. [20-22]. For different models have been derived results which disagree with each other. For example, Refs. [20] argue for an essential increase of the bulk viscosity at the critical point, contrary to the results of [21]. Lattice QCD calculations [23] applicable to the case of the sQGP demonstrate that the bulk viscosity is much smaller than the shear viscosity.

It is expected that at large baryon densities and not too high temperatures the hadron-sQGP phase transition is of first-order [24,25]. Lattice results $[26,27]$ support this conclusion. Signatures of such a transition might manifest themselves in heavy-ion collisions in a broad interval of energies, like those available at SPS (CERN) and at future low energy campaign RHIC (Brookhaven), and FAIR (GSI) and NICA (JINR) facilities. One expects strangeness trapping and enhancement of the kaon multiplicity fluctuations [28], enhancement of the soft pion yield [29] and baryon number density fluctuations [30], as signals of the first-order phase transition. The dynamics of classical modes of the chiral condensate in presence of an expanding fluid of quarks was studied in [16] in terms of the $\sigma$-model. Quarks were treated in the framework of relativistic ideal hydrodynamics. It was indicated that significant density inhomogeneities appear around the critical point of the first-order phase transition. Ref. [25] argued that the fireball may linger longer in the vicinity of the critical point due to a divergence of susceptibilities, e.g., the specific heat. Refs. [29,15] paid attention to the critical slowing down that limits the growth of the $\sigma$-field correlation length in the vicinity of the critical point. Some models speculate about explosive freeze-out assuming an increase of the viscosity close to the critical point, see [31].

Another relevant phenomenon is the NGL first-order phase transition that manifests itself in heavy-ion collisions in the expansion stage at low densities [32]. Possible effects of super-cooled vapor and super-heated liquid phases, as well as those of the spinodal region, have been considered in [33,34]. The occurrence of a negative specific heat $\left(C_{P}\right)$ was reported, as the first experimental evidence of the liquid-gas phase transition in heavy-ion collision reactions [35]. The complete list of references is too long to be included here. For a review of this interesting topic, see [36].

Mixed (so-called pasta) phases may occur in systems with two or more conserved charges (e.g. electric and baryon charges) undergoing first-order phase transitions, see [37]. The physical origin of this phenomenon is based on the fact that the electric charge can be conserved globally rather than locally. The pasta (gas-liquid) phases appear in the inner crusts of neutron star. Besides, pasta phase may arise also in the interior regions of compact stars, 
provided the equation of state (EoS) allows for first-order phase transitions to the pion or the kaon condensate, or to quark matter [38]. In equilibrium, pasta is constructed of Wigner-Seitz cells. The conservation of the electric charge is assumed within each Wigner-Seitz cell. Each Wigner-Seitz cell contains a seed (nucleus) of one phase surrounded by matter of the another phase. Seeds are droplets for a small concentration of the new phase, and rods and slabs for higher concentrations. Only little is known how pasta phases are cooked dynamically, see $[3,39]$.

In this paper, we will describe the dynamics of first-order phase transitions by means of the standard system of equations of non-ideal non-relativistic hydrodynamics: the Navier-Stokes equation,

$$
\begin{aligned}
& \rho \partial_{t} u_{i}+\rho(\vec{u} \nabla) u_{i} \\
& =-\nabla_{i} P+\nabla_{k}\left\{\eta\left(\nabla_{k} u_{i}+\nabla_{i} u_{k}-\frac{2}{d} \delta_{i k} \operatorname{div} \vec{u}\right)+\zeta \delta_{i k} \operatorname{div} \vec{u}\right\},
\end{aligned}
$$

the continuity equation,

$$
\partial_{t} \rho+\operatorname{div}(\rho \vec{u})=0
$$

and the general equation for the heat transport,

$$
\begin{aligned}
& T\left[\frac{\partial s}{\partial t}+\operatorname{div}(s \vec{u})\right] \\
& =\operatorname{div}(\kappa \nabla T)+\eta\left(\nabla_{k} u_{i}+\nabla_{i} u_{k}-\frac{2}{d} \delta_{i k} \operatorname{div} \vec{u}\right)^{2}+\zeta(\operatorname{div} \vec{u})^{2} .
\end{aligned}
$$

Here $\rho=m n, m$ is the mass of the constituents, $n$ is the density of the conserving charge (e.g, the baryon charge); $P$ is the pressure; $\eta$ and $\zeta$ are the first (shear) and second (bulk) viscosities; $\vec{u}$ is the velocity of the element of the fluid; $S$ is the entropy, $s=d S / d V ; \kappa$ is the thermal conductivity; as before, $d$ is the dimensionality of space. We solve these equations numerically in two spatial dimensions, $d=2$, and analytically for arbitrary $d$ in the vicinity of the critical point. Then we perform estimations for the cases of the NGL and the hadron-sQGP phase transitions.

The paper is organized as follows. In sect. 2 we analytically treat the dynamics of a system in the vicinity of the critical point of the first-order phase transition. First we perform a reduction of the general system of equations of non-ideal hydrodynamics to equations for the order parameters. Then, in order to solve the problem analytically, we use a density expansion of the Landau free energy functional in the vicinity of the critical point and derive the equation of motion for the density variable in dimensionless units. We study 
evolution of density fluctuations in metastable regions: the super-heated liquid and the super-cooled vapor. Then we consider the evolution of the seed shape and study the dynamics of density fluctuations in the spinodal region. In sect. 3 we find numerical solutions of the general system of hydrodynamical equations (2) - (4) in two spatial dimensions and compare these numerical results with those of the analytical treatment. We also pay attention to the specific patterns, which are not seen in a simplified analytical formulation, such as the dependence of the time evolution of the seed on its shape and on the power of the inflow of the surrounding matter. To be specific, throughout numerical calculations and analytical estimations we use phenomenological modified Van der Waals $(\mathrm{mVW})$ EoS. Its expansion in the vicinity of the critical point is done in the Appendix A and implies validity of the mean field approximation. A modification of the VW EoS which we do does not change the universality class, being the same for many substances named the VW fluids. In Appendix $\mathrm{B}$ assuming thermal equilibrium we evaluate a fluctuation region. Although our consideration is very general, allowing for further applications of the results to the description of specific nuclear dynamics, in sect. 4 we specify the parameters first for a nuclear matter system undergoing a NGL phase transition and then for a system undergoing a hadron-to-sQGP phase transition. Sect. 5 formulates conclusions.

Throughout the paper we use units $\hbar=c=1$. The results of this paper are briefly summarized in the letter [40].

\section{Small overcriticality}

\subsection{Reduction of equations of nonideal hydrodynamics to equations for the order parameter}

Assume that EoS allows for a first-order phase transition and conditions are such that the system is somewhere in the vicinity of the critical point. In this case pressure isotherm as function of the density has a convex-concave shape, as for the Van der Waals EoS.

Further in order to construct hydrodynamical description we need an expression for a thermodynamical potential depending on appropriate thermodynamical variables in each space-time point. Working in $(T, \rho)$ variables one may use relation between the pressure $P$ and the Helmholtz free energy $F$ :

$$
P=\left.\rho(\delta[F(T, \rho)] / \delta \rho)\right|_{T}
$$

If one wanted to work in $(s, \rho)$ variables, one could use that 


$$
P=\left.\rho(\delta[E(s, \rho)] / \delta \rho)\right|_{S}, \quad T=\left.\rho^{-1}(\delta[E(s, \rho)] / \delta s)\right|_{\rho},
$$

where $E(S, V)$ is the energy. These expressions generalize standard thermodynamical relations $P=-\left.(\partial F(T, V) / \partial V)\right|_{T}=-\left.(\partial E(S, V) / \partial V)\right|_{S}, T=$ $\left.(\partial E(S, V) / \partial S)\right|_{V}$ to the slightly spatially inhomogeneous configurations.

To treat the problem analytically let us expand quantities, entering EoS and equations of hydrodynamics, near some reference point $\left(T_{\mathrm{r}}, \rho_{\mathrm{r}}\right)$ on the curve $P(T, \rho)$ (or $\left(s_{\mathrm{r}}, \rho_{\mathrm{r}}\right)$ on the curve $P(s, \rho)$, respectively), where $\left(T_{\mathrm{r}}, \rho_{\mathrm{r}}\right)$ are assumed to be close to the values in the critical point, i.e. $0<T_{c r}-T_{\mathrm{r}} \ll T_{c r}$, $0<\left(\rho_{\text {liq }}^{\mathrm{MC}}-\rho_{\text {gas }}^{\mathrm{MC}}\right) / \rho_{\mathrm{r}} \ll 1$. It is convenient to take $\rho_{\mathrm{r}}$ satisfying the condition $\left.\left(\partial^{2} P(\rho, T) / \partial \rho^{2}\right)\right|_{\mathrm{r}}=0$, or, as an alternative, $\rho_{\mathrm{r}}=\frac{1}{2}\left(\rho_{\text {liq }}^{\mathrm{MC}}+\rho_{\text {gas }}^{\mathrm{MC}}\right)$ can be chosen, where $\rho_{\text {liq }}^{\mathrm{MC}}$ and $\rho_{\text {gas }}^{\mathrm{MC}}$ are densities at the liquid-gas equilibrium $\left(\mu_{\text {liq }}=\mu_{\text {gas }}=\mu_{\mathrm{MC}}, \mu\right.$ is the chemical potential) determined by the Maxwell construction (equal squares on the plot $\left.P(1 / \rho)\right|_{T}$, cut off by the horizontal line $P=$ const). Further to be specific we will take $T_{\mathrm{r}}=T_{c r}, \rho_{\mathrm{r}}=\rho_{c r}$. Then the above mentioned condition $\left.\left(\partial^{2} P(T, \rho) / \partial \rho^{2}\right)\right|_{\mathrm{r}}=0$ is fulfilled. In case of a $\mathrm{mVW}$ EoS, which we exploit in this paper, all necessary explicit expressions are presented in Appendix A.

We will consider evolution of fluctuations. These are, e.g., seeds of one phase in another phase and fluctuations like waves. Seeds can be of different shapes. Simplest forms of seeds in $d=3$ spatial dimensions are spherical droplets and bubbles, rods and slabs. Also seeds can have more peculiar shapes.

Since the evolution of collective modes is slower than that for microscopic modes, we further consider small velocities $\vec{u}(t, \vec{r})$ of the growth/damping of the density and temperature fluctuations. Thus we linearize hydrodynamical equations in the velocity " $u$ " and in the density $\delta \rho=\rho-\rho_{\mathrm{r}}$ and temperature $\delta T=T-T_{\mathrm{r}}$ variables. Introducing auxiliary variable $z=\operatorname{div} \vec{u}$ and applying operator "div" to both sides of the Navier-Stokes equation we obtain $[2,4]$ :

$$
\rho_{\mathrm{r}}(\partial z / \partial t)=-\Delta\left[\delta P-\left(\widetilde{d} \eta_{\mathrm{r}}+\zeta_{\mathrm{r}}\right) z\right], \quad \widetilde{d}=2(d-1) / d .
$$

Continuity equation becomes

$$
(\partial \delta \rho / \partial t)=-\rho_{\mathrm{r}} z
$$

Replacement of $z$ into Eq. (7) produces [4]

$$
\frac{\partial^{2} \delta \rho}{\partial t^{2}}=\Delta\left[\left.\rho_{\mathrm{r}} \frac{\delta[F(T, \delta \rho)]}{\delta(\delta \rho)}\right|_{T}+C+\rho_{\mathrm{r}}^{-1}\left(\widetilde{d} \eta_{\mathrm{r}}+\zeta_{\mathrm{r}}\right) \frac{\partial \delta \rho}{\partial t}\right]
$$

where we expressed the pressure in terms of the free energy in $T, \rho$ variables. We added an additional constant term $C$ in square brackets which will be 
specified below. Obviously $\Delta C=0$. Note that for $\delta \rho>0\left(\rho>\rho_{c r}\right)$ we deal with one phase and for $\delta \rho<0\left(\rho<\rho_{c r}\right)$, with another phase. Transport coefficients can differ in those phases. To simplify the problem we assume a smooth density and temperature dependence of transport coefficients at the transition through the critical point although in a narrow fluctuation region some of these quantities could have a singular behavior. We ignore this complication since such a temperature region is narrow and since it may take a long time to develop these singularities.

Equation for the density should be supplemented with the equation for the entropy, which in case of slow evolution of the seed acquires the form

$$
T_{\mathrm{r}}(\partial s / \partial t)=c_{V, \mathrm{r}}(\partial \delta T / \partial t)=\kappa_{\mathrm{r}} \Delta \delta T
$$

where $\eta_{\mathrm{r}}, \zeta_{\mathrm{r}}$ and $\kappa_{\mathrm{r}}$ are the kinetic coefficients taken at $T=T_{\mathrm{r}}$ and $\rho=\rho_{\mathrm{r}}$.

In terms of $s, \rho$ variables Eq. (9) reads

$$
\frac{\partial^{2} \delta \rho}{\partial t^{2}}=\Delta\left[\left.\rho_{\mathrm{r}} \frac{\delta[E(s, \delta \rho)]}{\delta(\delta \rho)}\right|_{s}+\rho_{\mathrm{r}}^{-1}\left(\widetilde{d}_{\eta_{\mathrm{r}}}+\zeta_{\mathrm{r}}\right) \frac{\partial \delta \rho}{\partial t}\right]
$$

and Eq. (10) acquires the form of the equation for the conserving order parameter, also known as Cahn-Hilliard equation [41]:

$$
T_{\mathrm{r}}(\partial s / \partial t)=\left.\kappa_{\mathrm{r}} \Delta(\delta[E(s, \rho)] / \delta s)\right|_{\rho}
$$

We should note that Eq. (9) differs from that is usually exploited in the framework of the phenomenological Landau approach, see (1), and from equations used for the description of the dynamics of first-order phase transitions in heavy-ion collisions, e.g. see [42-44,29], and in relativistic astrophysical problems [45]. Difference with Eq. (1) disappears, if one sets zero the square bracketed term in the r.h.s. of (9). Then Eq. (9) becomes

$$
\rho_{\mathrm{r}}^{-1}\left(\widetilde{d} \eta_{\mathrm{r}}+\zeta_{\mathrm{r}}\right) \frac{\partial \delta \rho}{\partial t}=\left.\rho_{\mathrm{r}} \frac{\delta[F(T, \delta \rho)]}{\delta(\delta \rho)}\right|_{T}+C=\left.\rho_{\mathrm{r}} \frac{\delta\left[F_{L}(T, \delta \rho)\right]}{\delta(\delta \rho)}\right|_{T} .
$$

From the first glance, such a reduction procedure is legitimate, if space-time gradients are small. However for a seed, being prepared in a fluctuation at $t=0$ with a distribution $\delta \rho(t=0, \vec{r})=\delta \rho(0, \vec{r})$, the condition $\left.\frac{\partial \delta \rho(t, \vec{r})}{\partial t}\right|_{t=0} \simeq 0$ should also be fulfilled. Otherwise there appears a positive kinetic energy contribution. Probability of such fluctuations should be suppressed. On the other hand, two initial conditions cannot be simultaneously fulfilled, if equation contains time derivatives of the first-order only. Thus there exists an initial stage 
of the dynamics of phase transitions ( $\left.t \lesssim t_{\text {init }}\right)$, which is not described by the standard Landau equation, see Eq. (1) or (13), being broadly exploited in condensed matter physics. R.h.s. of (13) presents thermodynamical force driving the system to the final (equilibrium) state. This force should become zero for $t \rightarrow \infty$, i.e. when the system reaches the final $(f)$ equilibrium state. Therefore $C=-P_{f}$. Thus instead of the Helmholtz free energy we may introduce the Landau free energy requiring that

$$
\left.\rho_{\mathrm{r}} \frac{\delta\left[F_{L}(T, \delta \rho)\right]}{\delta(\delta \rho)}\right|_{T}=P-P_{f}=0
$$

in the final equilibrium state.

Let the time scale for the relaxation of the density, following Eq. (9), is $t_{\rho}$ and the time scale for the relaxation of the entropy/temperature, following (10), is $t_{T}$. The latter quantity is estimated as

$$
t_{T}=R^{2} c_{V, \mathrm{r}} / \kappa_{\mathrm{r}} .
$$

Thus $t_{T}$ grows $\propto R^{2}$ with increase of the size of the seed $R$. On the other hand, following Eq. (9) $t_{\rho} \propto R$ (we show below that a seed of rather large size grows with constant velocity). Evolution of the seed is governed by the slowest mode. Thus, dynamics of seeds with sizes $R<R_{\mathrm{fog}}\left(\right.$ for $t_{T}(R)<t_{\rho}(R)$ ) is controlled by Eq. (9) for the density. Here $R_{\text {fog }}$ is the typical seed size at which $t_{\rho}=t_{T}$. For seeds with sizes $R>R_{\text {fog }}, t_{T} \propto R^{2}$ exceeds $t_{\rho} \propto R$ and growth of seeds is slown down. Thereby, number of seeds with the size $R \sim R_{\text {fog }}$ grows with time. If conditions are such that $\kappa$ is sufficiently large, $t_{T}$ exceeds $t_{\rho}$ only for seeds of rather large sizes. At terrestrial conditions, when growing droplet in the cloud becomes sufficiently large and heavy, it falls down under the action of the gravity. Falling down, the seed causes an avalanche of secondary droplets, if there are accumulated already many droplets of the size $R \sim R_{\mathrm{fog}}$. Thus solving Eqs. (9), (10) we are able to describe such a phenomenon as rain, provided gravitational forces are incorporated. Contrary, if $\kappa$ is sufficiently small, $t_{T}$ exceeds $t_{\rho}$ already for seeds of rather small sizes (at terrestrial conditions, it may occur when gravity is not yet efficient). Since for $R>R_{\text {fog }}$ growth of seeds is slowing down and thus number of seeds with the size $R \sim R_{\text {fog }}$ is increasing with time, there appears the fog.

Note that seeds of the new phase are produced in the old phase owing to short-scale fluctuations. The latter fluctuations are not incorporated in above hydrodynamical equations describing by the mean field variables. Contributions of short-scale fluctuations can be simulated by a random force induced in Eqs. (9), (10) with the help of the $\delta$-correlated source terms, cf. [2]. Being produced owing these source terms, large scale fluctuations (seeds) evolve in time following hydrodynamical equations. 
2.2 EoS in the vicinity of the critical point and equation for the density in dimensionless units

Consider evolution in $d$-dimensional space of seeds in case when the heat transport is not yet efficient and the dynamics is controlled by Eq. (9) for the density variable. For the sake of simplicity let us use a convenient parameterization of the Landau free energy, i.e. the generating functional in $\delta \rho, \delta T$ variables, which variation in $\delta \rho$ produces equation of motion

$$
\delta F_{L}=\int \frac{d^{3} x}{\rho_{\mathrm{r}}}\left[\frac{c[\nabla(\delta \rho)]^{2}}{2}+\frac{\lambda(\delta \rho)^{4}}{4}-\frac{\lambda v^{2}(\delta \rho)^{2}}{2}-\epsilon \delta \rho\right],
$$

where $\delta F_{L}=F_{L}[T, \rho]-F_{L}\left[T_{\mathrm{r}}, \rho_{\mathrm{r}}\right]$, that allows for the first-order phase transition. Here coefficients $c>0, \lambda>0$ are some functions of $T_{\mathrm{r}}$ and $\rho_{\mathrm{r}}, v$ and $\epsilon$ are yet functions of $\delta T$ and $|\epsilon| \ll \lambda v^{3}$. As we have mentioned, for convenience we choose $T_{\mathrm{r}}=T_{c r}, \rho_{\mathrm{r}}=\rho_{c r}$. For the $\mathrm{mVW}$ EoS, that we further exploit in our numerical calculations, see Appendix A, the value $v^{2}$ diminishes towards the critical point as $v^{2} \propto\left(T_{c r}-T\right)$.

For slightly inhomogeneous configurations the pressure can be expressed as

$$
\begin{aligned}
\delta P & =P-P\left(T_{\mathrm{r}}, \rho_{\mathrm{r}}\right) \\
& \left.\simeq \frac{\partial P}{\partial T}\right|_{T_{\mathrm{r}}, \rho_{\mathrm{r}}} \delta T+\left.\frac{1}{2} \frac{\partial^{2} P}{\partial T^{2}}\right|_{T_{\mathrm{r}}, \rho_{\mathrm{r}}}(\delta T)^{2}-\lambda v^{2} \delta \rho+\lambda(\delta \rho)^{3}-c \Delta \delta \rho .
\end{aligned}
$$

Here we used expansion of $\delta P$ near $T_{\mathrm{r}}, \rho_{\mathrm{r}}$ and Eqs. (14), (16). Thus

$$
\begin{aligned}
\epsilon & =P_{f}-P\left(T_{\mathrm{r}}, \rho_{\mathrm{r}}\right)-\left.\frac{\partial P}{\partial T}\right|_{T_{\mathrm{r}}, \rho_{\mathrm{r}}} \delta T-\left.\frac{1}{2} \frac{\partial^{2} P}{\partial T^{2}}\right|_{T_{\mathrm{r}}, \rho_{\mathrm{r}}}(\delta T)^{2}-\ldots \\
& \simeq P_{f}-P_{\mathrm{MC}} \simeq n_{c r}\left(\mu_{i n}-\mu_{f}\right)
\end{aligned}
$$

$\mu_{i n}$ and $\mu_{f}$ are the chemical potentials of the initial and final configurations (at fixed $P$ and $T$ ). For the $\mathrm{mVW}$ EoS the pressure expansion in $\delta T, \delta n$ near $T_{c r}$, $n_{c r}$ is performed in Appendix A. Then maximum value $\epsilon^{\max }=P^{\max }-P_{\mathrm{MC}} \simeq$ $n_{c r}\left(\mu^{\max }-\mu_{\mathrm{MC}}\right) \propto\left(T_{c r}-T\right)^{3 / 2}$, where $P^{\max }$ and the chemical potential $\mu^{\max }$ correspond to the state where $P(n)$ has local maximum $P^{\max }$, and $P_{\mathrm{MC}}, \mu_{\mathrm{MC}}$ are quantities on the Maxwell construction.

The Landau free energy density $\delta \mathcal{F}_{\text {rel }}=\delta \mathcal{F}_{L} / \mathcal{F}_{L}\left(T_{c r}, \rho_{c r}\right)$ and the value $\delta P_{\text {rel }}=$ $\left.\rho_{\mathrm{r}} \frac{\delta\left[F_{L}(T, \delta \rho)\right]}{\delta(\delta \rho)}\right|_{T} / P\left(T_{\mathrm{r}}, \rho_{\mathrm{r}}\right)$, for $T_{\mathrm{r}}=T_{c r}, \rho_{\mathrm{r}}=\rho_{c r}$, for spatially homogeneous configurations constructed following Eq. (16), as functions of the density $\delta \rho$, are schematically shown in Fig. 1 left and right, respectively. For $\epsilon>0$ (solid lines) the liquid state is stable and the gas state is metastable, and for $\epsilon<0$ 

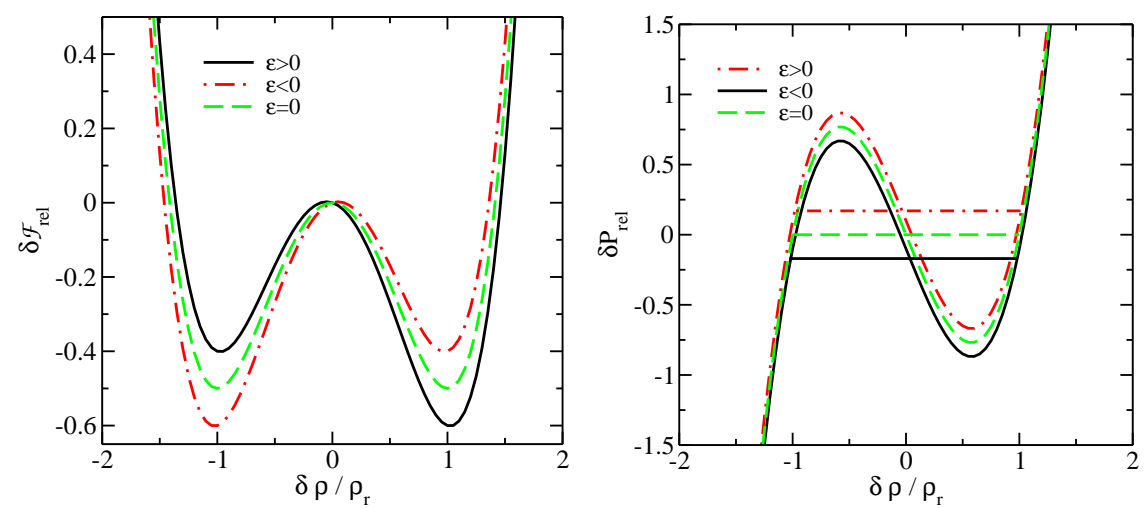

Fig. 1. The Landau free energy density $\delta \mathcal{F}_{\text {rel }}=\delta \mathcal{F}_{L} / \mathcal{F}_{L}\left(T_{c r}, \rho_{c r}\right)$ and the value $\delta P_{\text {rel }}=\left.\rho_{\mathrm{cr}} \frac{\delta\left[F_{L}(T, \delta \rho)\right]}{\delta(\delta \rho)}\right|_{T} / P\left(T_{c r}, \rho_{c r}\right)$ for uniform matter, as functions of the order parameter $\delta \rho$ for the EoS determined by Eq. (16). Dash horizontal line $(\epsilon=0)$ in the right panel shows Maxwell construction.

(dash-dotted lines) the liquid state is metastable, whereas the gas state is stable. For $\epsilon=0$ two minima of the Landau free energy coincide and correspond to the Maxwell construction on the curve $\delta P(1 / \rho)$ (shown by horizontal lines in the plot $\delta P(\delta \rho)$ in the right panel). Using Eq. (17), we rewrite Eq. (9) as

$$
-\frac{\partial^{2} \delta \rho}{\partial t^{2}}=\Delta\left[c \Delta \delta \rho+\lambda v^{2} \delta \rho-\lambda(\delta \rho)^{3}+\epsilon-\rho_{\mathrm{r}}^{-1}\left(\widetilde{d}_{\eta_{\mathrm{r}}}+\zeta_{\mathrm{r}}\right) \frac{\partial \delta \rho}{\partial t}\right]
$$

In dimensionless variables $\delta \rho=v \psi, \xi_{i}=x_{i} / l, i=1, \cdots, d, \tau=t / t_{0}$, Eq. (18) is simplified as

$$
\begin{aligned}
& -\beta \frac{\partial^{2} \psi}{\partial \tau^{2}}=\Delta_{\xi}\left(\Delta_{\xi} \psi+2 \psi\left(1-\psi^{2}\right)+\widetilde{\epsilon}-\frac{\partial \psi}{\partial \tau}\right) \\
& l=\left(\frac{2 c}{\lambda v^{2}}\right)^{1 / 2}, t_{0}=\frac{2\left(\widetilde{d} \eta_{\mathrm{r}}+\zeta_{\mathrm{r}}\right)}{\lambda v^{2} \rho_{\mathrm{r}}}, \widetilde{\epsilon}=\frac{2 \epsilon}{\lambda v^{3}}, \beta=\frac{c \rho_{\mathrm{r}}^{2}}{\left(\widetilde{d} \eta_{\mathrm{r}}+\zeta_{\mathrm{r}}\right)^{2}} .
\end{aligned}
$$

Eq. (19) is the key equation for our subsequent analysis. For $\rho_{\mathrm{r}}=\rho_{c r}, T_{\mathrm{r}}=T_{c r}$ taken, as the reference point, and for the VW fluids (see Appendix A) parameters demonstrate the following temperature dependence near the critical point: $v \propto|\delta T|^{1 / 2}, \epsilon \propto(\delta T)^{3 / 2}\left(\epsilon^{\max }=\sqrt{3} n_{c r}|\delta T|^{3 / 2} / T_{c r}^{1 / 2}, \widetilde{\epsilon}^{\max }=4 /(3 \sqrt{3})\right)$, and $l \propto|\delta T|^{-1 / 2}$ and $t_{0} \propto|\delta T|^{-1}$. The latter value shows that relaxation processes in the vicinity of the phase transition critical point prove to be very slow. This means that close to the critical point it is, indeed, legitimate to describe the phase transition dynamics in the framework of the non-relativistic approximation, as we do.

Note that values $c, \lambda, v, \epsilon$ in Eq. (16) are purely phenomenological coefficients. Introducing new variables $\lambda^{\prime}=\lambda m^{2}, v^{\prime}=v / m, \eta^{\prime}=\eta / m, \zeta^{\prime}=\zeta / m, \epsilon^{\prime}=\epsilon / m$ 
one could exclude, e.g., dependence on the mass of the constituent $m$ from all dynamical characteristics of the system. The value $m$ is not well defined quantity in case of the quark system. In new variables pressure and the Landau free energy are proportional to $m$, whereas all parameters entering Eq. (19) do not already depend on $m$.

As we have mentioned, Ref. [25] expressed an opinion that, if at some incident energy the trajectory passes the vicinity of the critical point, the system may linger longer in this region due to strong thermodynamical fluctuations resulting in the divergence of susceptibilities, that may reflect on observables. Contrary, we argue that fluctuation effects of the vicinity of the critical point in heavy-ion collisions can hardly be pronounced, since all relevant processes are proved to be frozen for $\delta T \rightarrow 0$, while the system passes this region during a finite time.

As one can see from Eq. (19), the dynamics of the phase transition is governed by single combination $(\widetilde{d} \eta+\zeta)$ of shear and bulk viscosities, cf. [43]. This observation might be interesting in connection with discussions of the particular effects of shear and bulk viscosities in sQGP, see [12].

The Landau free energy (16), being expressed in dimensionless variables, becomes

$$
\delta F_{L}=\frac{l^{3}}{\rho_{\mathrm{r}}} \frac{\lambda v^{4}}{2} \int d^{3} \xi\left[\frac{\left(\nabla_{\xi} \psi\right)^{2}+\left(\psi^{2}-1\right)^{2}}{2}-\tilde{\epsilon} \psi\right]-\frac{\lambda v^{4}}{4 \rho_{\mathrm{r}}} \int d^{3} x
$$

There exist homogeneous stationary solutions of Eq. (19):

$$
\psi \simeq \pm 1+\widetilde{\epsilon} / 4, \quad|\widetilde{\epsilon}| / 4 \ll 1
$$

corresponding to the Landau free energy density

$$
\delta \mathcal{F}_{L} \simeq-\frac{\lambda v^{4}}{4 \rho_{\mathrm{r}}} \mp \frac{\epsilon v}{\rho_{\mathrm{r}}}
$$

Thus for $\tilde{\epsilon}>0$ the upper sign solution describes stable liquid and the lower sign solution circumscribes super-cooled vapor, see Fig. 1. For $\widetilde{\epsilon}<0$ the lower sign solution describes stable gas and the upper sign solution circumscribes super-heated liquid. 


\subsection{Dynamics of seeds in metastable and in stable surroundings, for $t_{\rho} \gg t_{T}$}

\subsubsection{Seed density profile}

In $d=3$ space one deals with configurations of different symmetries, e.g., with spherically symmetric solutions $\left(d_{\text {sol }}=3\right.$, droplets/bubbles), axially symmetric solutions $\left(d_{\text {sol }}=2\right.$, liquid and gas rods) and one-dimensional solutions $\left(d_{\text {sol }}=1\right.$, liquid and gas slabs $)$. To describe mentioned configurations we search two-phase solution of Eq. (19) in the form, see [2-4],

$$
\psi \simeq \mp \tanh \left[\xi-\xi_{0}(\tau)\right]+\tilde{\epsilon} / 4
$$

$\xi=\xi_{1}$ for kinks (walls separating metastable and stable phases), $\xi=\sqrt{\xi_{1}^{2}+\xi_{2}^{2}}$, for rods, and $\xi=\sqrt{\xi_{1}^{2}+\xi_{2}^{2}+\xi_{3}^{2}}$, for droplets/bubbles. For $\widetilde{\epsilon}>0$ upper sign solution describes evolution of droplets (or rods and kinks of liquid phase) in a metastable super-cooled vapor medium. The lower sign solution circumscribes evolution of bubbles (or rods and kinks of the gas phase) in a stable liquid medium. For $\widetilde{\epsilon}<0$ the upper sign solution describes evolution of droplets in a stable gas medium, whereas the lower sign solution circumscribes then bubbles in a metastable super-heated liquid medium. From the kink solution for $\xi_{0} \gg 1$ (thin wall) one can easily construct the solution for the slab $\left(-\xi_{0}<\xi<\xi_{0}\right)$ :

$$
\psi \simeq \mp \operatorname{sgn} \xi \tanh \left[\xi-\operatorname{sgn} \xi \cdot \xi_{0}(\tau)\right]+\widetilde{\epsilon} / 4
$$

The boundary layer has the length $\left|\xi-\xi_{0}(\tau)\right| \sim 1$. Outside this layer corrections to homogeneous solutions are exponentially small. Considering motion of the boundary for $\xi_{0}(\tau) \gg 1$, we may put $\xi \simeq \xi_{0}(\tau)$ in $(23)$, (24). Then keeping only linear terms in $\epsilon$ in Eq. (19) we arrive at equation for $\xi_{0}(\tau)$,

$$
\frac{\beta}{2} \frac{d^{2} \xi_{0}}{d \tau^{2}}= \pm \frac{3}{2} \widetilde{\epsilon}-\frac{d_{\mathrm{sol}}-1}{\xi_{0}(\tau)}-\frac{d \xi_{0}}{d \tau}
$$

\subsubsection{Volume and surface contributions to the Landau free energy}

Substituting (24) in (20), subtracting infinite constant term and supposing, as we have used above $\xi_{0}(\tau) \gg 1$, we obtain

$$
\delta F_{L}\left[\xi_{0}\right]=\frac{2 \pi^{3 / 2} \Lambda^{3-d_{\mathrm{sol}}} \lambda v^{4} l^{d_{\mathrm{sol}}}}{\Gamma\left(d_{\mathrm{sol}} / 2\right) \Gamma\left(1+\left(3-d_{\mathrm{sol}}\right) / 2\right) \rho_{\mathrm{r}}}\left[\mp \frac{1}{d_{\mathrm{sol}}} \widetilde{\epsilon} \xi_{0}^{d_{\mathrm{sol}}}+\frac{2}{3} \xi_{0}^{d_{\mathrm{sol}}-1}\right],
$$

$2 \Lambda$ is the diameter, height of cylinder and the length of the squared plate for $d_{\text {sol }}=3,2$ and 1 , respectively; $\Gamma$ is the Euler $\Gamma$-function. The first term in (26) 
is the volume term and the second one is the surface contribution, $\delta F_{L \text {,surf }}$. At fixed volume in $d=3$ space, the surface contribution for droplets/bubbles is smaller than for rods and slabs. Thereby, if a seed prepared in a fluctuation is initially non-spherical, it acquires spherical form with passage of time (see discussion in subsection 2.4 below). The surface term is $\delta F_{L \text {,surf }} \equiv \sigma S, S$ is the surface of the seed, $\sigma$ is the surface tension. The gradient term in (16) is as follows

$$
\delta F_{L, \text { surf }}^{\mathrm{grad}}=\frac{1}{2 \rho_{\mathrm{r}}} c \int(\nabla \rho)^{2} d V=\frac{v^{2} c l}{2 \rho_{\mathrm{r}}} \int\left(\nabla_{\xi} \psi\right)^{2} d^{3} \xi=\frac{2 v^{2} c}{3 l \rho_{\mathrm{r}}} S=\frac{1}{2} \delta F_{L, \text { surf }}
$$

Now we are able to express the surface tension through parameters of the EoS. For the mVW EoS, see Appendix A, we find

$$
\sigma=\sigma_{0}|\delta \mathcal{T}|^{3 / 2}, \quad \sigma_{0}^{2}=32 m n_{c r}^{2} T_{c r} c ; \quad l=\frac{\sigma_{0}}{6 T_{c r} n_{c r}|\delta \mathcal{T}|^{1 / 2}}
$$

$\delta \mathcal{T}=\left(T-T_{c r}\right) / T_{c r}$

\subsubsection{Role of the viscosity and surface tension}

There are only two dimensionless parameters in Eqs. (19), (25), $\widetilde{\epsilon}$ and $\beta$. Parameter $\tilde{\epsilon}$ shows the difference in the Landau free energies of metastable and stable states, see Eqs. (22) and (23). Dynamics is controlled by the parameter $\beta$, which enters together with the second derivative in time. This parameter can be expressed in terms of the surface tension and the viscosity as

$$
\beta=\left(32 T_{c r}\right)^{-1}\left[\widetilde{d}_{\eta_{\mathrm{r}}}+\zeta_{\mathrm{r}}\right]^{-2} \sigma_{0}^{2} m
$$

The larger viscosity and the smaller surface tension, the effectively more viscous is the fluidity of seeds. For $\beta \ll 1$ one deals with the regime of effectively viscous fluid and at $\beta \gg 1$, with the regime of perfect fluid. Note that $\beta$ does not explicitly depend on the $n_{c r}$. As is shown experimentally [46], the ability of liquid domains to coalesce is controlled by an interplay between the surface tension and the viscosity. Parameter $\beta$, which we introduced, is responsible for such an interplay.

\subsubsection{Critical radius}

For the case of a metastable seed prepared in a stable surrounding, both terms in the Landau free energy $(26)$ are positive (at $d_{\text {sol }} \neq 1$ ). Thereby such seeds should shrink. For stable seeds developing in metastable surrounding the first (volume) term is negative. Therefore in this case there exists a critical 
size of the seed, which can be found by minimization of (26) in $\xi_{0}$ : $\xi_{0}^{c r}=$ $2\left(d_{\text {sol }}-1\right) /(3|\widetilde{\epsilon}|)$. For the mVW EoS, see Appendix A, the condition $\xi_{0}^{c r} \gg 1$, that we exploited deriving Eq. (25), is fulfilled for $|\widetilde{\epsilon}| \ll \widetilde{\epsilon}^{\max }$. The closer initial state to the Maxwell construction, the smaller is $|\widetilde{\epsilon}|$.

The Landau free energy (26) decreases with decrease of the droplet/bubble (or rod) size, provided $\xi_{0}(\tau)<\xi_{0}^{c r}$. Such seeds of the new phase, being produced, are dissolved with passage of time. For $\xi_{0}(\tau)>\xi_{0}^{c r}$ the Landau free energy decreases with increase of the seed size, that explains growth of overcritical droplets/bubbles and rods of the stable phase in the metastable medium.

In dimensional units the critical size of the droplet/bubble or rod becomes

$$
\begin{aligned}
R_{c r} & =\frac{\left(d_{\mathrm{sol}}-1\right) v^{2} \sqrt{2 c \lambda}}{3|\epsilon|} \\
R_{c r}\left(\gamma \epsilon^{\text {max }}\right) & =\frac{\sigma_{0}}{2 \sqrt{3} \gamma n_{c r} T_{c r}|\delta \mathcal{T}|^{1 / 2}},
\end{aligned}
$$

where the latter equality is valid for the mVW EoS, see Appendix A, and we put $|\epsilon|=\gamma \epsilon^{\max }$, where constant $0 \leq \gamma \leq 1$ characterizes deviation of $|\epsilon|$ from $\epsilon^{\max }$. The surface tension parameter $\sigma_{0}$ is given by Eq. (28). The smaller $|\epsilon|$, the larger is the critical radius of the droplet/bubble (or the rod) growing into the new phase. For $\epsilon \rightarrow 0$ (Maxwell construction) $R_{c r} \rightarrow \infty$.

Slabs of the stable phase, being placed in a metastable medium, grow independently of what was the value of their initial size $(R \gtrsim l)$.

\subsubsection{Probability of fluctuation}

The probability of the initial density fluctuation $\psi(\tau=0)$ of the size $\xi \simeq$ $\xi_{0}(\tau=0)$ (in dimensionless units), see $(24)$, is as follows

$$
W \sim e^{-\delta F_{L}\left[\xi_{0}(\tau=0)\right] / T}
$$

for $\xi_{0}(\tau=0)<\xi_{0}^{c r}$. For $\xi_{0}(\tau=0)>\xi_{0}^{c r}$ (unstable region) $\delta F_{L}$ decreases with the growth of $\xi_{0}$, however Eq. (31) does not hold anymore just indicating that these fluctuations may grow. Certainly, correctly calculated probability of appearance of a fluctuation with the size $\xi_{0}(\tau=0)>\xi_{0}^{c r}$ should decrease with increase of $\xi_{0}(\tau=0)$. Being produced in random processes, seeds evolve then according Eq. (25).

Two comments are in order. First, the profile (24) describes only one density distribution among various possible configurations, which can be produced in fluctuations. We should consider fluctuations involving many particles with 
the density $\rho \simeq \rho_{\text {liq }}$ in the interior region and $\rho \simeq \rho_{\text {gas }}$ in the exterior (provided liquid is stable phase). In difference with other configurations, the overcritical droplet described by solution (24) gains in the Landau free energy and conserves the form of the boundary layer, $\tanh (x)$, with time. Fluctuations with a different shape of the boundary layer reach $\tanh (x)$ - like shape after passage of a time, $\sim t_{\text {rec }}$, necessary for a reconstruction of the density profile. Second, various fluctuations may have distinct velocities $\frac{d \xi_{0}}{d \tau}$ of the seed boundary, the latter being specified as the point, where $\frac{\mathrm{d}^{2} \rho}{d \xi^{2}}=0$. However fluctuations containing a large number of particles, which we are interested in, can be cooked with not a negligible probability only provided the velocity $\frac{d \xi_{0}}{d \tau}$ is zero or very small $(\ll|\widetilde{\epsilon}|)$. Otherwise the Landau free energy in Eq. (31) (being $\propto|\widetilde{\epsilon}| \ll 1$, see $(22)$ ) would acquire essential positive contribution that would greatly diminish probability for the occurrence of such a configuration. Fluctuations with initially zero or very small values $\frac{d \xi_{0}}{d \tau}$, but with other density distributions in the surface layer compared to (24), will acquire a finite value of the velocity $\frac{d \xi_{0}}{d \tau}$ at the seed boundary after passage of a reconstruction time, $\sim t_{\text {rec }}$, when the density profile reaches the form of the profile given by Eq. (24). For $t \gg t_{\text {rec }}$ we may simulate all these cases with the profile of Eq. (24), assuming different values of $\frac{d \xi_{0}}{d \tau}$ at $\tau \sim \tau_{\text {rec }}$.

\subsubsection{Dynamics of slabs, $d_{\mathrm{sol}}=1$}

Consider dynamics of a slab of the stable phase (region $0<|\xi|<\xi_{0}(\tau)$ ) placed in a metastable surrounding $\left(|\xi|>\xi_{0}(\tau)\right)$. In this case one can find general solution of Eq. (25). First, let us obtain solutions satisfying initial conditions $\xi_{0}(\tau=0)=\xi_{0}(0),\left.\frac{d \xi_{0}}{d \tau}\right|_{\tau=0}=0$, since appearance of such seeds in fluctuations is more probable than, if $\left.\frac{d \xi_{0}}{d \tau}\right|_{\tau=0}$ were nonzero. Moreover $\left.\frac{d \xi_{0}}{d \tau}\right|_{\tau=0}=0$, if the seed is formed near the boundary of the system (provided the boundary is flat). Solution of Eq. (25) acquires the form

$$
\xi_{0}(\tau)=\xi_{0}(0)+\frac{3}{2}|\widetilde{\epsilon}| \tau-\frac{3}{4}|\widetilde{\epsilon}| \beta[1-\exp (-2 \tau / \beta)]
$$

Thus slabs of an arbitrary initial size (for $\xi_{0} \gg 1$ ) grow with passage of time.

In dimensional units we obtain

$$
R(t)=R_{0}+u_{\text {asymp }} t-\frac{3 c^{1 / 2}|\epsilon| \beta}{2^{1 / 2} \lambda^{3 / 2} v^{4}}\left[1-\exp \left(-\frac{\lambda v^{2} m n_{c r} t}{\left(\widetilde{d} \eta_{\mathrm{r}}+\zeta_{\mathrm{r}}\right) \beta}\right)\right],
$$

where $2 R_{0}$ is an initial size of the slab and

$$
u_{\text {asymp }}=3|\epsilon| v^{-2} \sqrt{\beta /(2 \lambda)},
$$




$$
u_{\text {asymp }}\left(\gamma \epsilon^{\max }\right)=\gamma|\delta \mathcal{T}|^{1 / 2} \sqrt{3 \beta T_{c r} /(2 m)}
$$

$u_{\text {asymp }}$ is the velocity of the growth of the slab at large values of time, $t \gg t_{\text {init }}$. Second equality (34) is valid for the mVW EoS. Then

$$
\begin{aligned}
\frac{3 \gamma c^{1 / 2} \epsilon^{\max } \beta}{2^{1 / 2} \lambda^{3 / 2} v^{4}} & =\frac{\gamma \beta \sigma_{0}}{6 \sqrt{3} n_{c r} T_{c r}|\delta \mathcal{T}|^{1 / 2}}, \\
\frac{\lambda v^{2} m n_{c r}}{\left(\widetilde{d} \eta_{\mathrm{r}}+\zeta_{\mathrm{r}}\right) \beta} & =\frac{72 n_{c r} T_{c r}^{2}\left(\widetilde{d} \eta_{\mathrm{r}}+\zeta_{\mathrm{r}}\right)|\delta \mathcal{T}|}{\sigma_{0}^{2} m}
\end{aligned}
$$

One can distinguish two stages of the evolution: an initial stage, $t \lesssim t_{\text {init }}$ $\left(\tau \sim \tau_{\text {init }}=\beta\right.$ in dimensionless units), and a subsequent stage, $t \gg t_{\text {init }}$ (or $\tau \gg \tau_{\text {init }}$ in dimensionless units). The initial stage lasts

$$
\begin{aligned}
t \sim t_{\text {init }} & =\frac{2\left(\tilde{d} \eta_{\mathrm{r}}+\zeta_{\mathrm{r}}\right) \beta}{\lambda v^{2} m n_{c r}} \\
t_{\text {init }} & =\frac{8 \beta\left(\tilde{d} \eta_{\mathrm{r}}+\zeta_{\mathrm{r}}\right)}{9 T_{c r} n_{c r}|\delta \mathcal{T}|}=\frac{\sigma_{0}^{2} m}{36 n_{c r} T_{c r}^{2}\left(\widetilde{d} \eta_{\mathrm{r}}+\zeta_{\mathrm{r}}\right)|\delta \mathcal{T}|}
\end{aligned}
$$

Second line (36) is for the mVW EoS. For $t \ll t_{\text {init }}$ from (33) we obtain

$$
R(t) \simeq R_{0}+w t^{2} / 2, \quad w=\frac{3|\epsilon| \lambda^{1 / 2}}{\sqrt{2 c}}
$$

that corresponds to the growing of the slab with constant acceleration.

Another typical time scale is $t_{\rho}=R / u_{\text {asymp }}$, since for $t \gg t_{\text {init }}$ from (33) we obtain $R=R_{0}+u_{\text {asymp }} t$ that corresponds to the growth of the size of the slab with constant velocity. In the vicinity of the critical point for the mVW EoS the velocity $u_{\text {asymp }}\left(\gamma \epsilon^{\max }\right) \propto|\delta \mathcal{T}|^{1 / 2}$ and

$$
t_{\rho}=\frac{R}{u_{\text {asymp }}\left(\gamma \epsilon^{\text {max }}\right)} \propto R|\delta T|^{-1 / 2} .
$$

Thus time scales $t_{\text {init }} \propto|\delta \mathcal{T}|^{-1}$ and $t_{\rho} \propto R|\delta \mathcal{T}|^{-1 / 2}$ demonstrate that all processes freeze out at the critical point.

For a large viscosity the time scale $t_{\text {init }} \propto 1 /\left(\tilde{d} \eta_{\mathrm{r}}+\zeta_{\mathrm{r}}\right)$ is rather short (excluding the vicinity of the critical point) and the system rapidly reaches the asymptotic regime. Contrary, in case of an ideal liquid typical time scale $t_{\text {init }}$ is long and thus during a long time the size of the slab grows with acceleration. 
For the initial condition corresponding to a nonzero velocity of the seed boundary (at $\tau=\tau_{\text {rec }}$ ) we find

$$
\begin{aligned}
\xi_{0}(\tau) & =\xi_{0}\left(\tau_{\text {rec }}\right)+\frac{3}{2}|\widetilde{\epsilon}|\left(\tau-\tau_{\text {rec }}\right)-\frac{\beta}{2}\left(\frac{3}{2}|\widetilde{\epsilon}|-\left.\frac{d \xi_{0}}{d \tau}\right|_{\tau=\tau_{\text {rec }}}\right) \\
& \times\left[1-\exp \left(-2\left(\tau-\tau_{\text {rec }}\right) / \beta\right)\right]
\end{aligned}
$$

instead of (32).

Another comment is in order. From Eq. (8) using (24) and the condition that the hydrodynamical velocity (the velocity of the inflow of the surrounding matter) $u_{\text {inflow }}=0$ deeply inside the slab, we find

$$
u_{\text {inflow }}(t)=-l t_{0}^{-1}\left(d \xi_{0} / d \tau\right)\left[\operatorname{sgn} \xi \tanh \left(\xi-\operatorname{sgn} \xi \cdot \xi_{0}\right)+1\right]
$$

At the right boundary of the slab $u_{\text {inflow }}\left(R=R_{0}(t)\right)=-l t_{0}{ }^{-1}\left(d \xi_{0} / d \tau\right)=$ $-d R(t) / d t$. For $R \gg R_{0}(t)$ at large $t, u_{\text {inflow }}\left(R \gg R_{0}(t)\right)=-3 \widetilde{\epsilon} l t_{0}{ }^{-1}=$

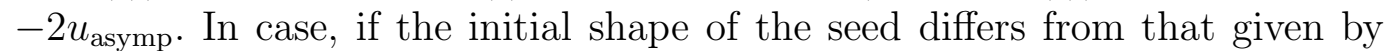
Eq. (24), the solution (40) is invalid for $t<t_{\text {rec }}$, where as before, $t_{\text {rec }}$ is the time scale of the reconstruction of the shape of the seed. From our numerical solutions below we will see that for effectively large viscosity, $\beta \ll 1$, the reconstruction period proves to be rather short and in opposite limit, $\beta \gg 1$, of an effectively small viscosity this time interval is very long.

\subsubsection{Initial stage of the seed evolution (for arbitrary $d_{\text {sol }}$ )}

Let us return to the consideration of the general case of an arbitrary value $d_{\text {sol }}$. As in previous subsection consider dynamics of stable seeds in a metastable surrounding. Then we may replace $\pm \widetilde{\epsilon}$ to $|\widetilde{\epsilon}|$ in Eq. (25). We search solution of Eq. (25) in the form of the Taylor expansion

$$
\xi_{0}(\tau)=a_{0}+a_{1} \tau+a_{2} \tau^{2}+a_{3} \tau^{3}+\ldots
$$

imposing initial conditions $\xi_{0}(\tau=0)=\xi_{0}(0),\left.\frac{d \xi_{0}(\tau)}{d \tau}\right|_{\tau=0}=0$. Then we find

$$
\xi_{0}(\tau)=\xi_{0}(0)+\frac{1}{2} w_{\tau} \tau^{2}\left(1-\frac{2 \tau}{3 \beta}\right)+\ldots, \tau \ll \min \left\{\tau_{\text {init }}, \sqrt{\xi_{0}(0) / w_{\tau}}\right\}
$$

where $w_{\tau}=2 \beta^{-1}\left[\frac{3}{2}|\widetilde{\epsilon}|-\frac{d_{\text {sol }}-1}{\xi_{0}(0)}\right]$. For slabs this result follows from the general solution (32) at $\tau \ll \tau_{\text {init }}$. In dimensional units Eq. (42) is rewritten as 


$$
R(t)=R_{0}+\frac{w t^{2}}{2}\left(1-\frac{2 t}{3 t_{0} \beta}\right)+\ldots, \quad t \ll \min \left\{t_{\text {init }},\left(R_{0} / w\right)^{1 / 2}\right\} .
$$

The acceleration

$$
w=d^{2} R / d t^{2}=\left(d_{\mathrm{sol}}-1\right) \lambda v^{2}\left(R_{0}-R_{c r}\right)\left(R_{c r} R_{0}\right)
$$

changes sign at $R_{0}=R_{c r}$. As we expected, seeds of undercritical size shrink with passage of time, whereas overcritical seeds grow. For seeds of a nearcritical size the process proceeds slowly $\left(w \propto|\delta \mathcal{T}|\left(R_{0}-R_{c r}\right) / R_{c r}^{2}\right)$. For undercritical seeds of a small size, $w \propto-|\delta \mathcal{T}| / R_{0}$. Note also that $w$ does not depend on the viscosity. For $d_{\text {sol }} \rightarrow 1$ from (43), (44) we recover result (37).

One could think that for slabs in case of perfect fluid Eq. (43) is valid for all times. Indeed, it follows from general solution (33) for $\beta \rightarrow \infty\left(t_{\text {init }} \rightarrow \infty\right.$ in this case). But motion with constant acceleration, see (44), becomes relativistic for large times. Deriving Eq. (19) from general system of hydrodynamical equations we dropped quadratic terms in the velocity. Thereby deriving (43) we additionally assumed that $t \ll \sqrt{R_{0} / w}$.

Notice, if we supposed that the velocity of the seed boundary were finite at $\tau=0$, we would obtain

$$
\xi_{0}(\tau)=\xi_{0}(0)+\left(\left.\frac{d \xi_{0}}{d \tau}\right|_{\tau=0}\right) \tau+\frac{1}{\beta}\left[\frac{3}{2}|\widetilde{\epsilon}|-\frac{d_{\mathrm{sol}}-1}{\xi_{0}(0)}-\left.\frac{d \xi_{0}}{d \tau}\right|_{\tau=0}\right] \tau^{2}+\ldots
$$

instead of Eq. (42), compare Eqs. (45) and (39) for $\tau_{\text {rec }}=0, \tau \ll \tau_{\text {init }}$.

\subsubsection{Late stage of the evolution ( $\left.t \gg t_{\text {init }}\right)$ of large seeds $\left(R(t) \gg R_{c r}\right)$}

Let $\beta \ll \xi_{0}^{2}$. Then we may drop the term $\frac{d^{2} \xi_{0}}{d \tau^{2}}$ in Eq. (25) and Eq. (25) simplifies as

$$
d \xi_{0}(\tau) / d \tau=\frac{3}{2}|\widetilde{\epsilon}|-\left(d_{\mathrm{sol}}-1\right) \xi_{0}^{-1}(\tau)
$$

Note that solutions of Eq. (46) do not satisfy necessary condition $\left.\frac{d \xi_{0}}{d \tau}\right|_{\tau=0}=0$, except for the case $\xi_{0}(0)=\xi_{0}^{c r}$. Therefore initial stage $\tau \lesssim \tau_{\text {init }}$ should be in any case described by more general Eq. (25), which is of the second order in time derivatives (see also discussion above in subsection 2.1). Thus besides the condition $\beta \ll \xi_{0}^{2}$ we should still require that $\tau \gg \tau_{\text {init }}=\beta$.

For $\xi_{0}(\tau) \gg \xi_{0}^{c r}$ (e.g. for initially overcritical droplets/bubbles and rods of a very large size, $\left.\xi_{0}(0) \gg \xi_{0}^{c r}\right)$ surface effects become unimportant. In this case 
one can neglect the term $\propto 1 / \xi_{0}$ in Eq. (46). Then for $\xi_{0}(\tau) \gg \xi_{0}^{c r}$ we arrive at the solution (32). Thus large seeds grow with constant velocity,

$$
\xi_{0}(\tau) \simeq \xi_{0}(0)+\frac{3}{2}|\widetilde{\epsilon}| \tau, \quad R(t) \simeq R_{0}+u_{\text {asymp }} t, \quad t \gg t_{\text {init }}
$$

The time scale for the growth of the seed of the size $R$ is

$$
\tau \sim \tau_{\rho}\left(\xi_{0}\right)=\frac{2 \xi_{0}}{3|\widetilde{\epsilon}|}, \quad t \sim t_{\rho}(R)=\frac{R}{u_{\text {asymp }}}
$$

for $t_{\rho} \gg t_{\text {init. }}$ Values $t_{\text {init }}$ and $t_{\rho}$ are the same as in (36) and (38). For the mVW EoS we estimate

$$
t_{\rho}\left(\gamma \epsilon^{\max }\right)=\frac{R}{u_{\text {asymp }}\left(\gamma \epsilon^{\text {max }}\right)}=\frac{4\left(\tilde{d} \eta_{\mathrm{r}}+\zeta_{\mathrm{r}}\right)}{3 \gamma^{2} n_{c r} T_{c r}|\delta \mathcal{T}|} \frac{R}{R_{c r}} \propto \gamma^{-2} R|\delta T|^{-1} .
$$

Both $t_{\text {init }}$ and $t_{\rho}\left(R_{c r}, \gamma \epsilon^{\max }\right)$ are $\propto|\delta T|^{-1}$.

From (36) and (49) we see that for the system near the critical point the time scale $\left.t_{\rho}\left(R_{c r}, \epsilon^{\max }\right)\right)$ is larger than $t_{\text {init }}$ for $\beta \ll 1$. In case of effectively small viscosity, $\beta \gg 1$, the time scale $t_{\text {init }}$ exceeds the value $t_{\rho}\left(R_{c r}, \gamma \epsilon^{\max }\right)$. Then the regime $t_{\rho}(R) \gg t_{\text {init }}$ is reached only for $R \gg R_{c r}\left(\gamma \epsilon^{\max }\right)$. Note that inequality $\beta \ll \xi_{0}^{2}$ is obviously fulfilled for all $\xi_{0}>\xi_{0}^{c r}$ for $t_{\rho}\left(R_{c r}, \gamma \epsilon^{\max }\right) \gg t_{\text {init }}$, i.e. $\tau_{\rho}\left(\xi_{0}^{c r}\right) \gg \beta$, since the latter inequality can be rewritten as $\beta \ll\left(\xi_{0}^{c r}\right)^{2}$.

\subsubsection{Evolution of seeds with nearcritical sizes (at $t \gg t_{\text {init }}$ )}

Let $d_{\text {sol }} \neq 1$ and we continue to consider evolution of seeds of a stable phase in a metastable surrounding. Consider a seed of initially nearcritical size, $\mid \xi_{0}(0)-$ $\xi_{0}^{c r} \mid \ll \xi_{0}^{c r}$, at an intermediate stage of its evolution, when the size of the seed still remains to be close to the critical one, $\left|\xi_{0}(\tau)-\xi_{0}(0)\right| \ll \xi_{0}^{c r}$. We assume that conditions $\tau \gg \tau_{\text {init }}=\beta$ and $\beta \ll \xi_{0}^{2}$ are again fulfilled. As we will see, evolution of the nearcritical seed proves to be very slow (cf. Eq. (52) below) and there exists a time interval, where these conditions are fulfilled. Solution of (46) is then as follows

$$
\begin{aligned}
& \xi_{0}(\tau)+\xi_{0}^{c r} \ln \frac{\xi_{0}(\tau)-\xi_{0}^{c r}}{\xi_{0}(0)-\xi_{0}^{c r}}=\xi_{0}(0)+\frac{\left(d_{\text {sol }}-1\right) \tau}{\xi_{0}^{c r}} \\
& d \xi_{0}(\tau) / d \tau=\left.\left(d \xi_{0}(\tau) / d \tau\right)\right|_{\text {n.cr }}=\left(d_{\text {sol }}-1\right)\left(\xi_{0}(\tau)-\xi_{0}^{c r}\right)\left[\xi_{0}^{c r} \xi_{0}(\tau)\right]^{-1}
\end{aligned}
$$

Using condition that the size of the seed is close to the critical size we find 


$$
\xi_{0}(\tau)=\xi_{0}(0)+\left(d_{\text {sol }}-1\right)\left[\xi_{0}^{c r} \xi_{0}(0)\right]^{-1}\left(\xi_{0}(0)-\xi_{0}^{c r}\right) \tau .
$$

In dimensional units the latter equation renders

$$
R(t)=R_{0}+u_{\text {n.cr }} t, \quad u_{\text {n.cr }}=\frac{\left(d_{\text {sol }}-1\right) c \rho_{\mathrm{r}}\left(R_{0}-R_{c r}\right)}{R_{0} R_{c r}\left(\widetilde{d} \eta_{\mathrm{r}}+\zeta_{\mathrm{r}}\right)} .
$$

As from Eq. (44), we see that seeds (droplets/bubbles and rods) of overcritical size grow, whereas seeds of undercritical size shrink. The velocity of the growth/shrinking of nearcritical seeds (for $d_{\text {sol }} \neq 1,\left|\xi_{0}(0)-\xi_{0}^{c r}\right| / \xi_{0}^{c r} \ll 1$ ) proves to be very low $\left(\propto\left|R_{0}-R_{c r}\right|\right)$. Typical time, when initially nearcritical seed still continues to be nearcritical, is

$$
t \sim t_{\mathrm{n} . \mathrm{cr}}=\frac{\left(R_{c r}\right)^{3}\left(\tilde{d}_{\eta_{\mathrm{r}}}+\zeta_{\mathrm{r}}\right)}{\left(d_{\mathrm{sol}}-1\right) c \rho_{\mathrm{r}}\left|R_{0}-R_{c r}\right|} \gg t_{\mathrm{init}}
$$

that justifies omitting of the term $\frac{d^{2} \xi_{0}}{d \tau^{2}}$, as we have done it. In the vicinity of the critical point for the mVW EoS, $t_{\text {n.cr }}\left(\gamma \epsilon^{\max }\right) \propto \gamma^{-3}\left|R_{0}-R_{c r}\right|^{-1}|\delta \mathcal{T}|^{-3 / 2}$. Thereby dynamics of seeds at this stage is very slow: $t_{\mathrm{n} . \mathrm{cr}} \gg\left(t_{\rho}\left(R_{c r}, \gamma \epsilon^{\max }\right), t_{\mathrm{init}}\right)$.

\subsubsection{Evolution of seeds of initially small size $\left(l \ll R_{0} \ll R_{c r}\right)$ at $t \gg t_{\text {init }}$}

For $\tau \gg \tau_{\text {init }}, \xi_{0}^{2} \gg \beta$, describing seeds of a small size $\left(1 \ll \xi_{0} \ll \xi_{0}^{c r}, d_{\text {sol }} \neq 1\right)$ we can drop the term $\propto \widetilde{\epsilon}$ in (46). Then solution satisfying initial condition $\xi_{0}(\tau=0)=\xi_{0}(0)$ acquires the form

$$
\xi_{0}(\tau) \simeq \sqrt{\xi_{0}^{2}(0)-2\left(d_{\mathrm{sol}}-1\right) \tau}, \quad \frac{d \xi_{0}(\tau)}{d \tau}=-\frac{\left(d_{\mathrm{sol}}-1\right)}{\sqrt{\xi_{0}^{2}(0)-2\left(d_{\mathrm{sol}}-1\right) \tau}}
$$

In dimensional units

$$
R(t) \simeq \sqrt{R_{0}^{2}-2\left(d_{\mathrm{sol}}-1\right) t l^{2} / t_{0}}
$$

For $\tau \gg \tau_{\text {init }}, \xi_{0}^{2}(0) \gg \beta$, i.e. for $t_{\text {init }} \ll t \ll t_{\text {dis }}$, from (55) we find

$$
R(t)=R_{0}+u_{\mathrm{dis}} t, \quad u_{\mathrm{dis}}=-\frac{\left(d_{\mathrm{sol}}-1\right) c \rho_{0}}{R_{0}\left(\widetilde{d} \eta_{\mathrm{r}}+\zeta_{\mathrm{r}}\right)} .
$$

The time scale of the dissolution of the initial fluctuation of a small size

$$
t_{\mathrm{dis}}=\frac{\left(\tilde{d} \eta_{\mathrm{r}}+\zeta_{\mathrm{r}}\right) R_{0}^{2}}{2\left(d_{\mathrm{sol}}-1\right) c \rho_{\mathrm{r}}}=\frac{16 n_{c r} T_{c r}\left(\tilde{d} \eta_{\mathrm{r}}+\zeta_{\mathrm{r}}\right) R_{0}^{2}}{\left(d_{\mathrm{sol}}-1\right) \sigma_{0}^{2}}
$$


(in dimensional units) proves to be $\propto R_{0}^{2}$. Thereby, fluctuations of sufficiently small sizes are easily produced and then they are rapidly dissolved. As we see from (55), (56), at $t \gtrsim t_{\text {init }}$ the speed of the dissolution first reaches a constant value and then the process is rapidly accelerated.

Note that solving the problem we assumed $\xi_{0} \gg 1$ and $\xi_{0}^{2}(0) \gg \beta$. Both conditions are satisfied at least in case of an effectively large viscosity, $\beta \ll 1$.

\subsubsection{Numerical solution of equation for the droplet/bubble boundary}

Peculiarities of different regimes, which we have described analytically in limit cases, are demonstrated by numerical solutions of Eq. (25) presented in Figs. 2 and 3 on example of the time dependence of dimensionless velocity $\frac{d \xi_{0}(\tau)}{d \tau}$. Insertions in Figs. 2 and 3 show evolution of seeds at small times.

Fig. 2 shows numerical solution of Eq. (25) for undercritical seeds. In the left panel we consider evolution of the seed of a small size $\left(\xi_{0}(0)=0.3 \xi_{c r}\right)$ and in the right panel, of the seed of a near-critical size $\left(\xi_{0}(0)=0.999 \xi_{0}^{c r}\right)$. We see that undercritical seeds are shrinking with time. For the seed of a sufficiently small size (left panel) shrinking process is rather fast. The larger viscosity (smaller $\beta$ ), the faster is the process. One distinguishes two stages of the process: an initial stage $\left(\tau \lesssim \tau_{\text {init }}=\beta\right)$, and a dissociation stage, $\tau_{\text {init }} \ll$ $\tau \lesssim \tau_{\text {dis }}$, see $(57)$. The latter stage is subdivided by two stages: $\tau \ll \tau_{\text {dis }}$ (characterizing by approximately constant velocity) and $\tau \sim \tau_{\text {dis }}$ (when the process is rapidly accelerated). The curve labeled as "approx" demonstrates analytical solution Eq. (54), being valid for $\tau \gg \tau_{\text {init }}$, that requires $\tau_{\text {dis }} \gg \tau_{\text {init }}$. The latter condition is not fulfilled for $\beta=10$ (effectively small viscosity), but it is fulfilled for $\beta=10^{-1}$ (effectively large viscosity). Deviation of the dash-dotted curve from the solid one for $\beta=10^{-1}$ is due to the fact that the ratio $\xi_{0}(0) / \xi_{0}^{c r}=0.3$ is not yet much less than unity, and $\xi_{0}(0) \simeq 4$ is not yet much larger than unity, whereas conditions $\xi_{0}(0) / \xi_{c r} \ll 1, \xi_{0}(0) \gg 1$ are required for validity of the analytical solution (54). We have checked this with various choices of parameters. Significant difference between the dash-dotted and the dash curve in case of a small effective viscosity (large $\beta$ ) is due to the fact that $\tau_{\text {init }}>\tau_{\text {dis }}$ in this case. Thus for an effectively small viscosity dissolution of the small size seed occurs at the time scale $\tau \sim \tau_{\text {init }}$.

For the nearcritical seed (see right panel of Fig. 2) one can distinguish three stages of the process: initial stage $\left(\tau \lesssim \tau_{\text {init }}=\beta\right)$; an intermediate stage, when the seed still continues to have nearcritical size, $\left(\xi_{0}^{c r}-\xi_{0}(\tau)\right) / \xi_{0}^{c r} \ll 1$ (it lasts very long, up to $\tau \lesssim 500$ ); and a short subsequent dissociation stage. Dependence on the viscosity (the value $\beta$ ) does not manifest itself at the intermediate stage. The "n.cr." arrow in Figure insertion shows the value of the velocity which follows from our analytical solution (51). 

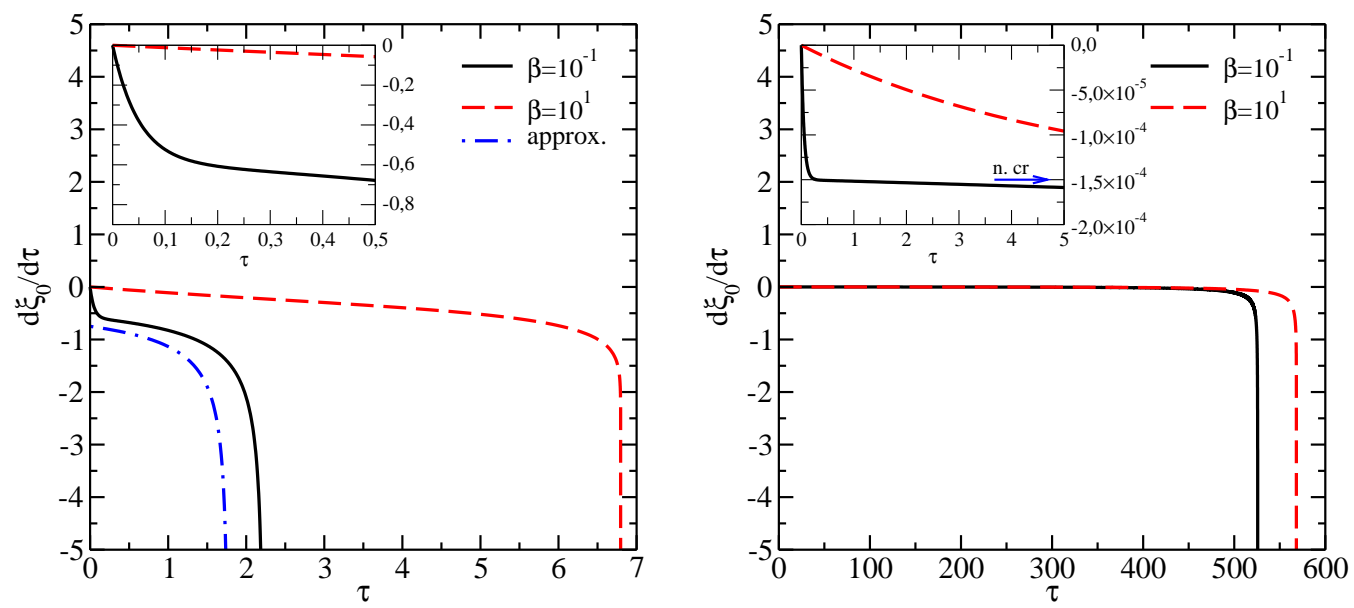

Fig. 2. Numerical solution of Eq. (25) for the undercritical droplet of initial size $\xi_{0}(0)=0.3 \xi_{0}^{c r}$ (left panel) and for the near-critical seed, $\xi_{0}(0)=0.999 \xi_{0}^{c r}$, (right panel) for effectively large viscosity (solid curves) and for effectively small viscosity (dash curves); $\widetilde{\epsilon}=0.1$. Dash-dotted line is analytical solution given by Eq. (54).
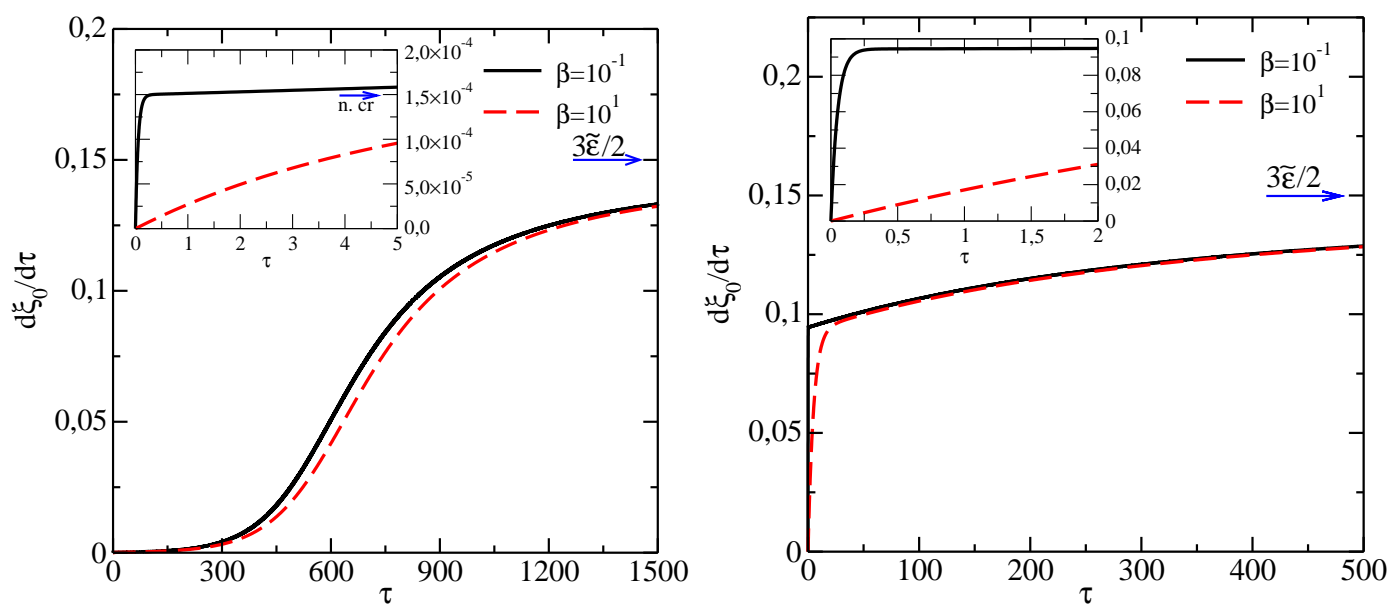

Fig. 3. Numerical solution of Eq. (25) for the overcritical droplet of initial size $\xi_{0}(0)=1.001 \xi_{0}^{c r}$ (left) and for $\xi_{0}(0)=1.1 \xi_{0}^{c r}$ (right) for two values of $\beta$. The arrow $3 \widetilde{\epsilon} / 2$ shows asymptotic value of the velocity (in dimensionless units).

In Fig. 3 we demonstrate numerical solution of Eq. (25) for overcritical seeds. We see that these seeds are growing with time. For initially nearcritical seed (left panel), there exist four stages of the process. Initial stage lasts a time $\tau \lesssim \tau_{\text {init }}=\beta$, see Figure insertion. Next is a prolonged stage, when $\left(\xi_{0}(\tau)-\right.$ $\left.\xi_{0}^{c r}\right) / \xi_{0}^{c r} \ll 1$ (velocity is almost constant for $\tau \lesssim 200$ ). Then there is a transition stage (for $\tau \lesssim 1500 \div 2000$ ), when $\left(\xi_{0}(\tau)-\xi_{0}^{c r}\right) / \xi_{0}^{c r} \sim 1$, and finally the asymptotic regime, when the velocity reaches new constant value $\frac{3}{2} \widetilde{\epsilon}$ (shown in Figure by arrow). On a so long time scale the memory about initial stage of the evolution (the latter depends on $\beta: \tau \lesssim \tau_{\text {init }}=\beta$ ) is lost: dash and solid curves coincide. Note however that in dimensional units asymptotic regime is 
achieved much faster in case of a more viscous fluid.

The right panel of Fig. 3 shows evolution of the overcritical seed for $\xi_{0}(0)=$ $1.1 \xi_{0}^{c r}$. The process proceeds faster, than for $\xi_{0}(0)=1.001 \xi_{0}^{c r}$, and in three stages. From initial stage through a transition stage the seed reaches asymptotic regime. Transition regime lasts very long.

As we see, presented numerical solutions of Eq. (25) support our simplified analytical considerations performed above.

\subsection{Evolution of the shape of seeds}

To be specific consider solutions in $d=3$ space. Majority of seeds, being produced in fluctuations, have near spherical form. Then their shape evolves with passage of time. To describe this evolution let us expand $\xi_{0}(\theta, \phi, \tau)$ in spherical functions:

$$
\xi_{0}=\sum_{l, m} \xi_{0}(l, m) Y_{l, m}(\theta, \phi)
$$

It is convenient to use shorthand notations $\xi_{0}(l=0, m)=\xi_{0}(l=0)$ and $\xi_{0}(l \neq$ $0, m)=\xi_{0 l}$. Further assume that the seed has almost spherical form, i.e. $\xi_{0 l} \ll$ 1. Using that $\Delta_{\xi}=\frac{\partial^{2}}{\partial \xi^{2}}+\frac{2}{\xi} \frac{\partial}{\partial \xi}+\frac{\hat{L}^{2}}{\xi^{2}}, \frac{\hat{L}^{2} \xi_{0 l}}{\xi^{2}}=\frac{l(l+1) \xi_{0 l}}{\xi^{2}}$, in the linear approximation in $\xi_{0 l}$ from Eq. (25) we obtain equation for the time evolution of the seed boundary (for $\left.\xi_{0 l}\right)$. It looks cumbersome. Dropping terms $\propto O\left(\frac{d \xi_{0}}{d \tau}, \frac{d^{2} \xi_{0}}{d \tau^{2}}\right)$ we are able to simplify equation as follows

$$
\begin{aligned}
\frac{1}{2} \frac{d^{2} \xi_{0 l}}{d \tau^{2}} & =\frac{(2-l(l+1)) \xi_{0 l}}{\widetilde{\beta} \xi_{0}^{2}(l=0, \tau)}-\frac{1}{\widetilde{\beta}} \frac{d \xi_{0 l}}{d \tau}, \quad l \geq 1 \\
\widetilde{\beta} & =\beta\left[1+\frac{1}{2} l(l+1) \xi_{0}^{-2}(l=0, \tau)\right]^{-1} .
\end{aligned}
$$

For $\xi_{0}^{2}(l=0, \tau) \gg l^{2}$, that we will further assume (just to deal with simpler expressions) one has $\widetilde{\beta} \simeq \beta$. The value $\xi_{0}(l=0, \tau)$ obeys Eq. (25). Undamped mode with $l=1$ describes the motion of the system as a whole.

\subsubsection{Initial stage of the evolution}

For $\tau \ll \min \left(\tau_{\text {init }}, \sqrt{\xi_{0}(0) / w_{\tau}}\right)$, see solution (42), Eq. (59) is further simplified as 


$$
\frac{1}{2} \beta \frac{d^{2} \xi_{0 l}}{d \tau^{2}}+\frac{d \xi_{0 l}}{d \tau}+\frac{(l(l+1)-2) \xi_{0 l}}{\xi_{0}^{2}(l=0, \tau=0)}=0 .
$$

Solution of this equation is as follows: $\xi_{0 l}=C_{1} e^{\lambda_{1} \tau}+C_{2} e^{\lambda_{2} \tau}$. Using initial condition $\left.\frac{d \xi_{0 l}}{d \tau}\right|_{\tau=0}=0$ one obtains $C_{1}=-\frac{\lambda_{2}}{\lambda_{1}-\lambda_{2}} \xi_{0 l}(0), C_{2}=\frac{\lambda_{1}}{\lambda_{1}-\lambda_{2}} \xi_{0 l}(0)$,

$$
\lambda_{1,2}=-\frac{1}{\beta} \pm \sqrt{\frac{1}{\beta^{2}}-\frac{2(l(l+1)-2)}{\beta \xi_{0}^{2}(l=0, \tau=0)}} .
$$

Thus we find two damping solutions. For

$$
1 \ll \xi_{0}(l=0, \tau=0)<\sqrt{2 \beta[l(l+1)-2]}, \quad l>1,
$$

besides damping there occur oscillations. Inequalities (62) are fulfilled for $\beta \gtrsim$ $\xi_{0}^{2}(l=0, \tau=0)$ (limit of effectively very small viscosity).

\subsubsection{Limit of an effectively small viscosity}

The time scale of oscillations is $\tau \sim \tau_{l}^{\text {id/osc }}=\left|\min \left(\lambda_{1,2}\right)\right|^{-1} \sim \sqrt{\beta} \xi_{0}(l=$ $0, \tau=0) \lesssim \tau_{\text {init }}$ provided inequality $(62)$ is fulfilled. Condition $\tau_{l}^{\text {id/osc }} \ll \tau_{\text {init }}$ is satisfied only for very large $\beta \gg|\widetilde{\epsilon}|^{-2}$ in case $\xi_{0}(l=0, \tau=0) \sim \xi_{0}^{c r}$ and for $\beta \gg 1$ for seeds of a small size $\xi_{0}(l=0, \tau=0) \gtrsim 1$. In dimensional units the time scale

$$
t_{l}^{\mathrm{id} / \mathrm{osc}} \simeq \frac{2 R_{0}}{3 \sqrt{[l(l+1)-2]}}\left(\frac{m}{T_{c r}|\delta \mathcal{T}|}\right)^{1 / 2} \lesssim t_{\text {init }}
$$

does not depend on the value of the viscosity.

Damping occurs at the time scale $\tau_{l}^{\text {id/damp }} \sim \beta$, that corresponds to

$$
t \sim t_{l}^{\text {id/damp }} \sim t_{\text {init }}
$$

in dimensional units. Thus the time scale for the reconstruction of the initial density profile of the seed (if initially it deviates only slightly from the profile Eq. (23)), is $t_{\text {rec }} \sim t_{l}^{\text {id/damp }} \sim t_{\text {init }}$ in this case.

\subsubsection{Limit of an effectively large viscosity}

For $\beta \ll 1 \quad$ (effectively large viscosity) the time scale $\min \left(\tau_{\text {init }}\right.$, $\left.\sqrt{\xi_{0}(l=0, \tau=0) / w_{\tau}}\right)$ is very short and for $\tau \gg \tau_{\text {init }}$ one can drop the term 
$\frac{d^{2} \xi_{0}(l=0)}{d \tau^{2}}$ in l.h.s. of Eq. (59). Then the latter equation is simplified as, cf. [2]:

$$
\frac{(2-l(l+1)) \xi_{0 l}}{\xi_{0}^{2}(l=0)}-\frac{d \xi_{0 l}}{d \tau}=0
$$

Its solution is as follows:

$$
\xi_{0}^{l}(\tau)=\xi_{0}^{l}(0) \exp \left\{\int_{0}^{\tau} d \tau^{\prime}[2-l(l+1)] \xi_{0}^{-2}\left(l=0, \tau^{\prime}\right)\right\} .
$$

All modes with $l>1$ prove to be damped. Thus an initially deformed seed acquires spherical shape with time. Typical time scale is

$$
\tau_{l}^{\eta}=\frac{\xi_{0}^{2}(l=0, \tau=0)}{[l(l+1)-2]}, \quad t_{l}^{\eta}=\frac{\rho_{c r} R_{0}^{2}}{\beta\left(\widetilde{d} \eta_{\mathrm{r}}+\zeta_{\mathrm{r}}\right)[l(l+1)-2]}, \quad l>1
$$

We find that $t_{l}^{\eta} \sim t_{\text {dis }}$ for not too high $l$ and for $\xi_{0}(l=0, \tau=0)<\xi_{0}^{c r}$, see Eq. (57). The larger $l$, the more rapid is the process. For $\xi_{0}(l=0, \tau=0)>\xi_{0}^{c r}$, $\xi_{0}(l=0, \tau=0)-\xi_{0}^{c r} \sim \xi_{0}^{c r}$ we obtain $t_{l}^{\eta} \sim t_{\rho}$.

\subsection{Dynamics of density fluctuations in spinodal region}

Assume that the system is driven to the spinodal region (region between the minimum and the maximum of $\left.\delta P(\delta \rho)\right|_{T}$ in Fig. 1 (right). In the spinodal region even fluctuations of an infinitesimal amplitude and size may grow. To demonstrate this assume that the density is such that the free energy $\delta F_{L}$ is close to its maximum $\left(\delta F_{L} \simeq 0\right)$. Then we may linearize Eq. (19) dropping $\psi^{3}$ term. Setting

$$
\psi=-\frac{\widetilde{\epsilon}}{2}+\operatorname{Re}\left\{\psi_{0} e^{\gamma_{\psi} \tau+i \vec{k} \vec{\xi}}\right\}
$$

where $\psi_{0}$ is an arbitrary but small real constant, we find two solutions of linearized Eq. (19),

$$
\gamma_{\psi}(k)=(2 \beta)^{-1}\left(-k^{2} \pm \sqrt{k^{4}(1-4 \beta)+8 \beta k^{2}}\right) .
$$

Growing modes correspond to the choice of " +"-sign and $k^{2}<2$. Most rapidly growing mode is described by the maximum value of $\gamma_{\psi}(k)$ and $k=k_{m}$ : 


$$
k_{m}^{2}=\frac{2 \sqrt{\beta}}{2 \sqrt{\beta}+1}, \quad \gamma_{\psi}\left(k_{m}\right)=\frac{2}{2 \sqrt{\beta}+1} .
$$

Thus the time scale for the growth of seeds in aerosol (spinodal region) is

$$
t_{\mathrm{aer}}=t_{0} / \gamma_{\psi}\left(k_{m}\right)
$$

Increase of the wave amplitude is stopped, when the matter is exhausted in the regions, where $\psi_{0} e^{\gamma_{\psi} \tau} \cos (\vec{k} \vec{\xi})<0$. It occurs for $\psi_{0} e^{\gamma_{\psi} \tau} \sim 1$. After that, seeds (regions $\psi_{0} e^{\gamma_{\psi} \tau} \cos (\vec{k} \vec{\xi})>0$ with typical size $\left.l / \gamma_{\psi}\right)$, for $\psi_{0} e^{\gamma_{\psi} \tau} \sim 1$, begin to expand and flow together, until the stable phase is cooked. With logarithmic accuracy the time scale of the formation of the aerosol-like state is estimated as $t_{\text {aer }}$, following Eq. (71). The well known phenomenon illustrating exponential growth of fluctuations in the spinodal region is the boiling of the champaign occurring, when one opens the bottle.

\subsubsection{Limit of an effectively large viscosity}

For effectively large viscosity $(\beta \ll 1)$ we get two solutions:

$$
\gamma_{\psi}^{(1)}(k) \simeq 2\left[1-k^{2} / 2-\beta k^{2}\left(2 / k^{2}-1\right)^{2}\right], \quad \gamma_{\psi}^{(2)}(k) \simeq-k^{2} / \beta
$$

for $k^{2} \gg 4 \beta$. For $k<\sqrt{2}, \gamma_{\psi}^{(1)}(k)$ describes growing mode. The mode $\gamma_{\psi}^{(2)}(k)$ is damped. The most rapidly increasing mode corresponds to the momentum $k_{m}=\sqrt{2} \beta^{1 / 4}($ see $(70))$, and $\gamma_{\psi}^{(1)}\left(k_{m}\right) \simeq 2$. The time scale characterizing growth of this mode is

$$
t_{\mathrm{aer}}^{\eta} \sim t_{0} / 2=\left(\widetilde{d} \eta_{\mathrm{r}}+\zeta_{\mathrm{r}}\right)\left[\lambda v^{2} \rho_{\mathrm{r}}\right]^{-1}=4\left(\widetilde{d} \eta_{\mathrm{r}}+\zeta_{\mathrm{r}}\right)\left[9 n_{c r} T_{c r}|\delta \mathcal{T}|\right]^{-1}
$$

Second equality in (73) is valid for the mVW EoS. The larger the viscosity and the smaller $-\delta \mathcal{T}$, the slower is the time evolution. Since $k_{m}=\sqrt{2} \beta^{1 / 4}$, the size scale of seeds is

$$
R_{\mathrm{aer}}^{\eta} \simeq l /\left(\sqrt{2} \beta^{1 / 4}\right)
$$

Thus the size of seeds in aerosol increases with increase of the viscosity. For $k^{2}>2$ both modes are damped.

\subsubsection{Limit of an effectively small viscosity}

In case of effectively small viscosity $(\beta \gg 1)$ we get 


$$
\gamma_{\psi}(k) \simeq \pm k \sqrt{2 / \beta} \sqrt{1-k^{2} / 2}
$$

and the maximum value $\left(\gamma_{\psi}^{\max }\right)$ corresponding to $k_{m}=1$ is determined by

$$
\gamma_{\psi}^{\max }(1)=1 / \sqrt{\beta}
$$

As we see, the time scale characterizing the growing mode,

$$
t_{\mathrm{aer}}^{\mathrm{id}} \sim t_{0} / \gamma_{\psi}=2 c^{1 / 2} /\left(\lambda v^{2}\right) \gg t_{0}
$$

does not depend on the viscosity in this limit. For the mVW EoS one has $v^{2} \propto|\delta T|$ and $t_{\text {aer }}^{\text {id }} \propto 1 /|\delta T|$. Evolution then is slow near the critical point. Conditions for the validity of the non-relativistic approximation, which we have used, are fulfilled. For $t>t_{\mathrm{aer}}^{\mathrm{id}}$, growth of modes becomes exponentially fast, see (68). Since $k_{m}=1$, the typical size of seeds in aerosol is

$$
R_{\mathrm{aer}}^{\mathrm{id}} \simeq l, \quad R_{\mathrm{aer}}^{\mathrm{id}}<R_{\mathrm{aer}}^{\eta}
$$

Modes with $k^{2}>2$ correspond to oscillations. Such fluctuations do not grow to the stable phase.

\subsection{Evolution of the density fluctuations in the system close to equilibrium}

Assuming that $\psi$ is close to its new equilibrium value, $\psi_{e q} \simeq \pm 1+\tilde{\epsilon} / 4$, we put $\psi=\psi_{e q}+\delta \psi$ in Eq. (19) and linearize the latter equation in $\delta \psi$ :

$$
-\beta \frac{\partial^{2} \delta \psi}{\partial \tau^{2}}=\Delta_{\xi}\left(\Delta_{\xi} \delta \psi-4 \delta \psi-\frac{\partial \delta \psi}{\partial \tau}\right)
$$

Setting

$$
\delta \psi=\operatorname{Re}\left\{\psi_{0} e^{\gamma_{\phi} \tau+i \vec{k} \vec{\xi}}\right\}
$$

where $\psi_{0}$ is an arbitrary but small real constant, we find

$$
\gamma_{\psi}(k)=(2 \beta)^{-1}\left(-k^{2} \pm \sqrt{k^{4}(1-4 \beta)-16 \beta k^{2}}\right)
$$

Dependence on the quantity $\tilde{\epsilon}$ disappears from (79) and (81). 


\subsubsection{Limit of an effectively large viscosity}

In case of effectively large viscosity $(\beta \ll 1)$ and for $k^{2}>16 \beta$ there are only damped solutions. For $k^{2} \gg 8 \beta$ :

$$
\gamma_{\psi}^{(1)}(k) \simeq-\left(k^{2}+4\right), \quad \gamma_{\psi}^{(2)}(k) \simeq-k^{2} / \beta .
$$

Fluctuations with large $k$ rapidly dissolve with time. Existence of long living short-wave excitations is unlikely in the viscous medium. However there remain long-wave damped oscillations, for $k^{2}<16 \beta$.

\subsubsection{Limit of an effectively small viscosity}

In case of effectively small viscosity $(\beta \gg 1)$ we get

$$
\gamma_{\psi}(k)=-(2 \beta)^{-1}\left(k^{2} \pm 4 i \sqrt{\beta} k \sqrt{1+k^{2} / 4}\right),
$$

that corresponds to oscillating and slowly damped modes near the final equilibrium state, rather than to unstable modes. Since $t_{0} \sqrt{\beta}$ does not depend on the viscosity and $t_{0} \beta \rightarrow \infty$ for $\eta, \zeta \rightarrow 0$, in case of the ideal fluid rapid oscillations continue till the energy is transported to the surface of the system (the process is governed by the heat transport) or till the energy is radiated away in the course of direct reactions. Thus in case of effectively small viscosity the stable phase is covered by fine ripples during some rather long period of time.

\subsection{The Reynolds number and turbulence}

The transition between laminar and turbulent flows occurs, when the Reynolds number

$$
\operatorname{Re}=u \Lambda \rho_{f l} / \eta
$$

exceeds the critical Reynolds number $\operatorname{Re}_{c r}$, which is very large $(\gtrsim 1000)$. Here $u$ is the mean fluid velocity, $\Lambda$ is the characteristic diameter of the body embedded in the fluid and $\rho_{f l}$ is the density of the fluid. In particular problem of the growth of the droplet/bubble of the stable phase developing in the metastable surrounding, $u$ means the velocity of the growth of the seed, $\rho_{f l}$ is the density of the metastable phase ( $\simeq \rho_{c r}$ in case of small overcriticality) and $\Lambda=2 R$. Supposing $R=\alpha R_{c r}, \alpha=$ const, for the typical radius of the seed, taking $u$ from Eq. (34) and replacing these values in (84) we find 


$$
\operatorname{Re}=\frac{16}{3} \alpha \beta
$$

We see that for relevant values of $\alpha \lesssim 10$ factor Re may reach critical value only for unrealistically large values of the parameter $\beta$ (for a tiny effective viscosity). For values of $\beta$ and $\delta \mathcal{T}$ with which we are concerned, one has $\mathrm{Re} \ll \operatorname{Re}_{c r}$.

\section{Numerical integration of the system of hydrodynamical equa- tions in one and two spatial dimensions}

\subsection{Setup}

To diminish computing time we consider solutions in $d=2$ space. As is seen from analytical solutions presented above, qualitative description of the dynamics of the first-order phase transition remains similar for $d=3$ and $d=2$. E.g., Eq. (26) continues to hold for $d=2$, provided one replaces

$\delta F_{L}^{(d=3)}\left[d_{\text {sol }}=2 ; 1\right] /(2 L)$ to $\delta F_{L}^{(d=2)}\left[d_{\text {sol }}=2 ; 1\right]$. Further, we assume validity of the isothermal approximation neglecting the heat transport effects. In this case evolution is governed by Eqs. (2) and (3). This means that in the present paper we will not simulate the late stage of the evolution of fluctuations, when a fog-like state is formed.

To solve the problem we need to know EoS and transport coefficients. Since we will focus on demonstration of a qualitative behavior of the system undergoing the first-order phase transition, to avoid extra complications we will consider simplest case assuming that the viscosity does not depend on the density and the temperature. As we have mentioned in Introduction, for nuclear systems shear and bulk viscosities, as well as the heat conductivity, are poorly known and most probably the bulk viscosity is smaller than the shear one. Note that in analytical expressions, which we have derived, viscosities enter in combination $\left(\widetilde{d} \eta_{\mathrm{r}}+\zeta_{\mathrm{r}}\right)$. Thereby, and in order to diminish uncertainties we further put zero the bulk viscosity and vary the shear viscosity in broad limits.

The Landau free energy and the pressure of the uniform matter behave as shown in Fig. 1. Note that in general case the Helmloltz free energy density of the uniform matter does not produce two minima. Rather it fulfills the double-tangent construction (at values of densities corresponding to the Maxwell construction on the isotherm $P(1 / \rho))$. E.g., it is so for the original VW EoS and for the EoS of the relativistic mean field Walecka model. We use the mVW EoS, $P=f(T) P_{V W}$, see Appendix A. Extra function $f(T)$ is introduced for generality since temperature dependence of the purely VW 
EoS is too simple to describe behavior of nuclear matter. At constant $T$ for all $f(T)$ hydrodynamical descriptions are self-similar. E.g., the description of the dynamics in the framework of the original VW EoS can be obtained with the help of the scaling $t \rightarrow t \sqrt{f(T)}$ and $\left(\widetilde{d} \eta_{\mathrm{r}}+\zeta_{\mathrm{r}}\right) \rightarrow\left(\widetilde{d} \eta_{\mathrm{r}}+\zeta_{\mathrm{r}}\right) / \sqrt{f(T)}$, as it follows from Eq. (9). In our numerical calculations we specified $f(T)$ in order the Helmholtz free energy had two minima, as well as the Landau free energy. Such a modification is quite not necessary since hydrodynamical equations enters only gradient of the pressure, which in both cases has the same form. Moreover we use $f(T) \rightarrow 1$ for $T \rightarrow T_{c r}$. In this case all the results valid in the vicinity of the critical point do not depend on $f$ and are the same as for the original VW EoS. If we wanted to recover $f$-dependence for $f\left(T \rightarrow T_{c r}\right)=f_{0} \neq 1$ in the vicinity of the critical point, we could do it with help of the replacement $t_{0} \rightarrow t_{0} / f_{0}, \beta \rightarrow \beta f_{0}$ in Eq. (19).

In the present paper we consider dynamics of the phase transition at fixed pressure at the system boundary. In order to describe possible first-order phase transitions in heavy-ion collisions one still should take into account expansion of the fireball, see [47]. Moreover, one needs to use a more realistic EoS, e.g. that constructed in [48]. These problems will be considered in forthcoming publications.

The surface contribution to the pressure (the so called Laplace pressure) is taken into account in the low gradient approximation:

$$
P=P_{\mathrm{mVW}}-c \nabla^{2} \rho,
$$

with $\rho=m n$. The coefficient " $c$ " can be expressed in terms of the surface tension, see (28). We examine both one- and two-space dimensional solutions. In general case no assumptions about cylindrical symmetry are used. To illustrate the dynamics of the overcritical and undercritical seeds we consider a fluctuation in infinite matter in both spatial directions $x$ and $y$. Initial conditions correspond to a stable spot placed in the homogeneous metastable medium.

\subsection{Evolution of disks $\left(d_{\mathrm{sol}}=2\right)$ in $d=2$ spatial dimensions}

First consider dynamics of an initially static axial-symmetric seed with the conserved number density given by

$$
\rho(x, y ; t=0)=\rho_{\text {out }}+\left(\rho_{\text {in }}-\rho_{\text {out }}\right) \Theta\left(R_{0}-r\right), \quad r=\sqrt{x^{2}+y^{2}} .
$$

Such a seed would correspond to the rod in case $d=3$. Densities $\rho_{\text {in }}$ and $\rho_{\text {out }}$ are taken to be those in homogeneous phases. We call these configurations liquid or gas disks in dependence, if $\rho_{\text {in }}>\rho_{\text {out }}$ or vise versa. 

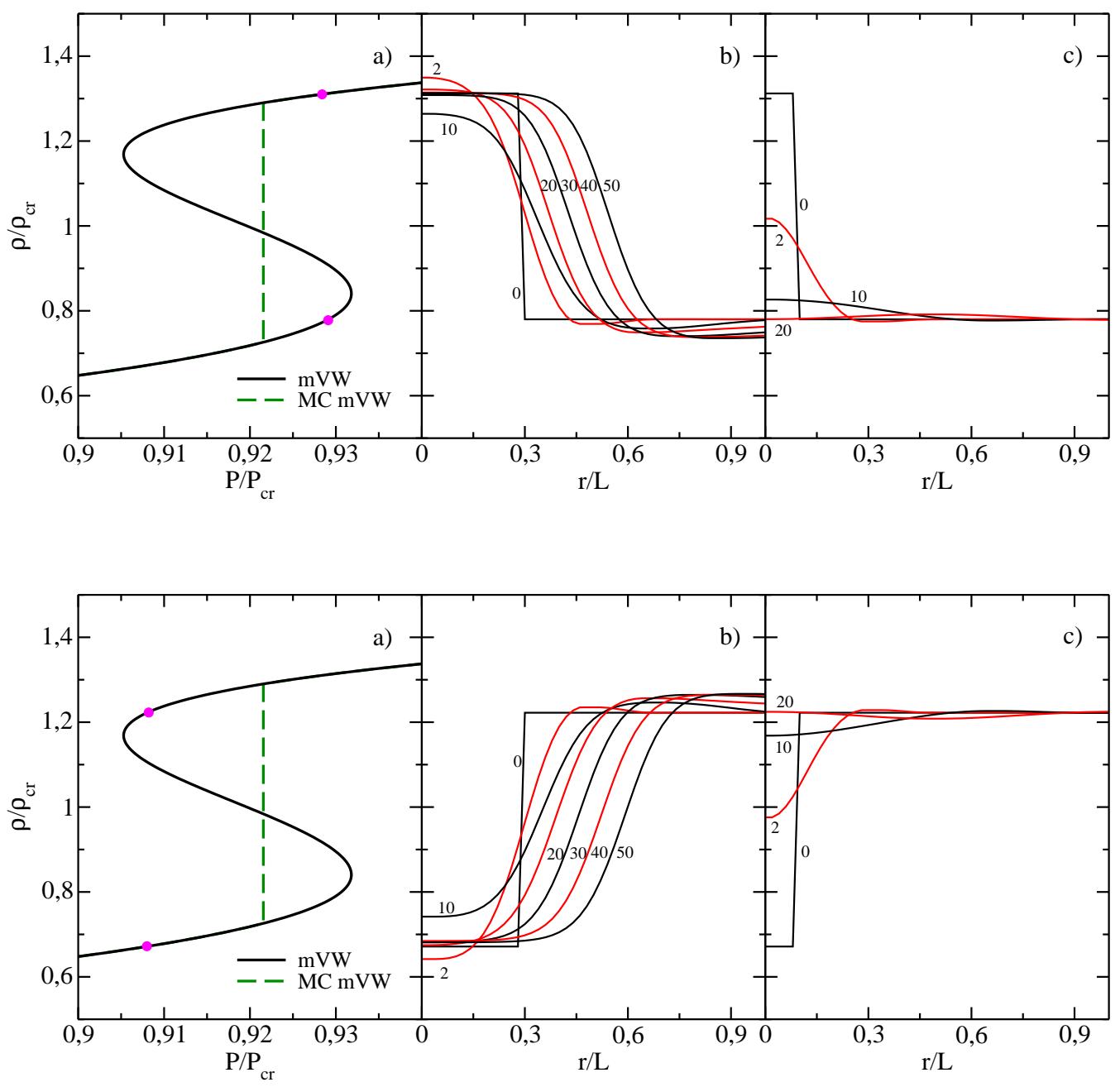

Fig. 4. Isotherm for the pressure as function of the density for mVW EoS, see Appendix A, with initial and final configurations shown by dots (left column). Dash vertical line corresponds to the Maxwell construction (MC) on the curve $P(1 / \rho)$. In upper panel initial state relates to stable liquid phase disk in metastable super-cooled gas and on lower panel it corresponds to the stable gas phase disk in metastable super-heated liquid. Middle column demonstrates time evolution of the density profiles for the overcritical liquid disk (upper panel) and gas disk (lower panel). Numbers near curves (in $L$ ) are time snapshots; $r=\sqrt{x^{2}+y^{2}},|\delta \mathcal{T}|=0.02$, $L=30 \mathrm{fm}$. Right column, the same for initially undercritical liquid or gas disks.

For $d=2$, expansion of the mVW EoS near the critical point is valid only for $|\delta \mathcal{T}| \ll 1 / 7$, see Appendix A, and analytical solution (23) is applicable for $|\delta \mathcal{T}| \ll 1 / 16\left(\xi_{0} \gg 1\right)$. Therefore we first take $T$ very close to the critical temperature, in order one could quantitatively compare results of computing with analytical expressions. Results are presented in Fig. 4 for $T / T_{c r}=0.98$. Parameters of EoS are chosen, as for the NGL phase transition: $T_{c r}=18.6 \mathrm{MeV}$, $n_{c r} / n_{\text {sat }}=0.42, n_{\text {sat }}=0.16 \mathrm{fm}^{3}$ is the nuclear saturation density. The configuration is computed for values of kinetic parameters chosen as $\eta \simeq 3.2 \mathrm{MeV} / \mathrm{fm}^{2}$ and $\beta \simeq 12.6$ (effectively small viscosity). For a large viscosity general behav- 

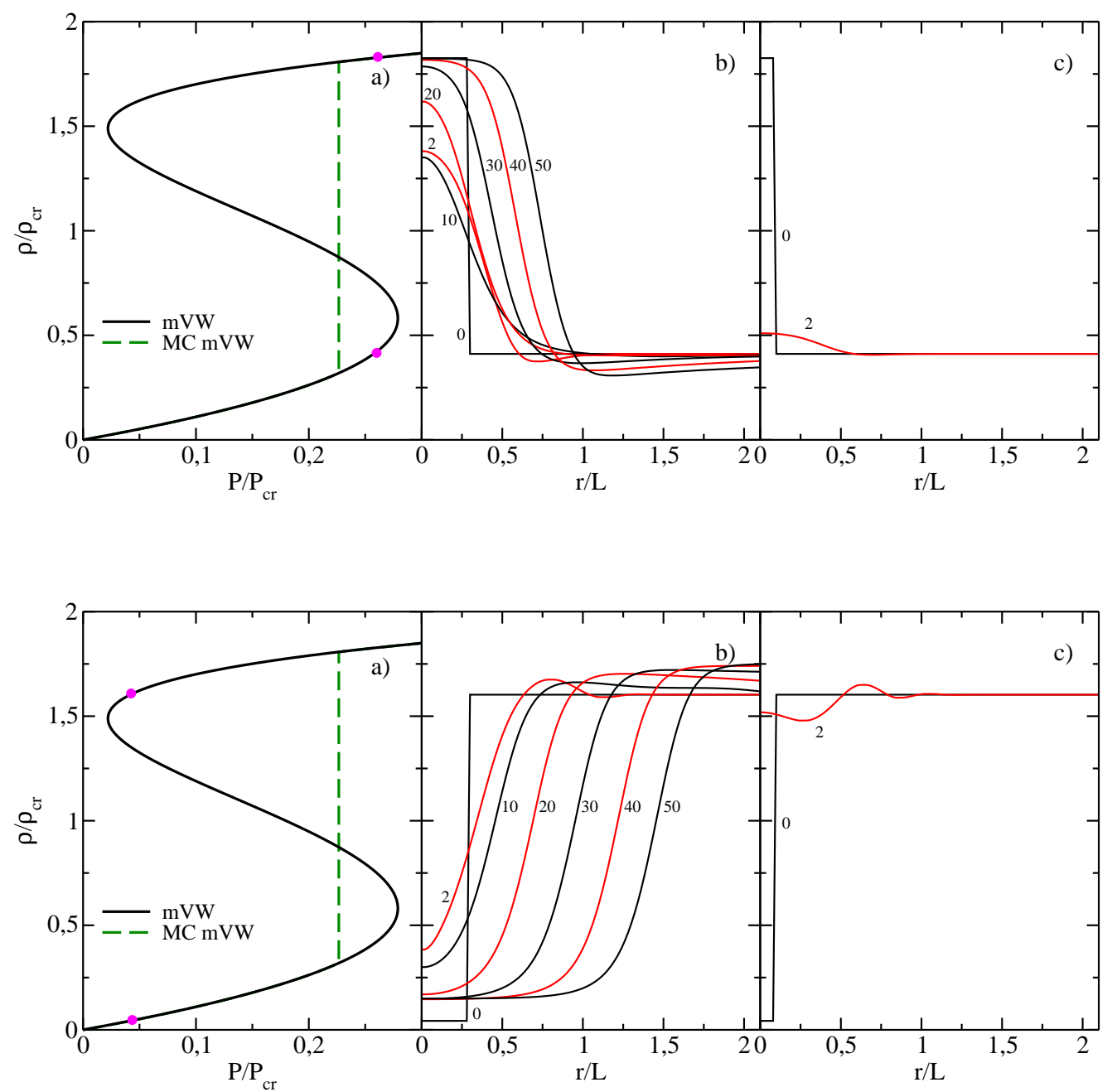

Fig. 5. The same as in Fig. 4 but for $|\delta \mathcal{T}|=0.15, L=5 \mathrm{fm}$.

ior of solutions remains the same but velocity of the seed evolution proves to be significantly smaller. Some peculiarities of the $\beta$ dependence will be illustrated in next figures. In the upper panel of Fig. 4 we demonstrate the time evolution of initially liquid disk and in the lower panel, of a gas disk. Left column demonstrates initial and final configurations on the curve $P(\rho)$. In the middle column we show dynamics of the initially overcritical seed $R_{0}=0.3 L>R_{c r} \simeq 0.2 L$ and in the right column, of the undercritical seed $R_{0}=0.1 L$, in units of a relevant length scale $L=30 \mathrm{fm}$. The time snapshots are shown by numbers near curves in units $L$ (i.e. 2 means $\Delta t=2 L$, etc.). We see from the middle column that in case $R_{0}>R_{c r}$ (in this example $R_{0} \simeq 1.5 R_{c r}$ ) disks slowly grow with time. The initially selected distribution (87) acquires tanh-like shape (see (23)) for $t \gtrsim 20 L \simeq 600 \mathrm{fm}$. This is very large time (although in dimensionless units it corresponds to a rather short time scale $\tau \gtrsim 6$, of the order of the duration of the initial stage, see the right panel of Fig. 3). As we see from the right column of Fig. 4, seeds of an initially small size $R_{0}<R_{c r}$ (in this example $R_{0} \simeq 0.5 R_{c r}$ ) dissolve for $t \gtrsim 20 L \simeq 600 \mathrm{fm}$. This value agrees 
with the total time of the shrinking process described by the dash curve in the left panel of Fig. 2. The typical time scale characterizing the dynamics is $t \sim t_{\text {init }} \gg t_{\text {dis }}$.

We have checked that the time evolution occurs in a line with above analytical consideration. The only difference is that in analytical treatment of the problem with initial distribution of the tanh- form, we do not get values $\rho<\rho_{\text {out }}$ for droplets and $\rho>\rho_{\text {out }}$ for bubbles. As follows from our numerical solution, due to infall of the surrounding matter to the disk surface during the shape reconstruction, the density decreases in the liquid disk neighborhood below the value of the density in the homogeneous metastable matter and it increases in the gas disk surrounding above the value of the density in the homogeneous metastable matter (see the middle column of Fig. 4).

Note that it is unlikely to find the system in heavy-ion collisions in so narrow vicinity of the critical point as we considered, $|\delta \mathcal{T}|=0.02$, since the typical time of the fireball expansion is much shorter than typical time of the evolution of fluctuations that we found. Therefore in Fig. 5 we also demonstrate the time evolution of disks for $T / T_{c r}=0.85$. For easier comparison with Fig. 4 initial and final configurations are selected at approximately the same deviations $\left(P-P_{\min }\right) /\left(P_{\max }-P_{\min }\right)$. In this Figure we take $L=5 \mathrm{fm}$, as the length unit. The system described by the VW EoS is already rather far from the critical point at $T / T_{c r}=0.85$. Indeed, for $T / T_{c r}>T_{\text {comp }} / T_{c r}=\frac{27}{32} \simeq 0.844$ there appears a region of negative pressures, that may cause some extra peculiarities in the processes under consideration. Different physical situations occurring for $T<T_{\text {comp }}$ were discussed in [33]. In this work we will avoid further discussion of the regime $T<T_{\text {comp }}$. Although for $T / T_{c r}=0.85$ the dynamics looks qualitatively the same, as in case demonstrated by Fig. 4, the critical radius proves to be significantly smaller $\left(R_{c r} \simeq 1 \mathrm{fm}\right.$ instead of $15 \mathrm{fm}$ in previous case) and the time scale characterizing the process is reduced. Therefore for $T / T_{c r}=0.85$ seeds evolve much faster compared to the case $T / T_{c r}=0.98$. For overcritical discs the initially selected distribution (87) acquires the tanh-like shape (see (23)) for $t \gtrsim(20 \div 40) L=100 \div 200 \mathrm{fm}$. The typical time scale is $t \sim t_{\text {init }}$, which in case $|\delta \mathcal{T}|=0.15$ is much shorter than in case $|\delta \mathcal{T}|=0.02$ considered above. Initial disks of a small size $\left(R_{0} \simeq 0.5 R_{c r}\right)$ almost disappear for $t \gtrsim 10 L=50 \mathrm{fm}$. In this case $t_{\text {init }} \sim t_{\text {dis. }}$. All these values of time scales are larger or of the order of the time scale characterizing the fireball expansion in low energy heavy-ion collisions.

We further varied parameters $T_{c r}, n_{c r}, \eta, \beta$ in broad limits (in the range relevant for the NGL phase transition) and checked that it does not change the qualitative picture presented in Figs. 4, 5. We demonstrate it in Fig. 6 showing the evolution of the disk surface with time for $R_{0}>R_{c r}$. The disk surface is specified as the boundary, where density achieves the critical value $\left(\rho=\rho_{c r}\right)$. In the left panel results are presented for $T / T_{c r}=0.98$. The velocity 

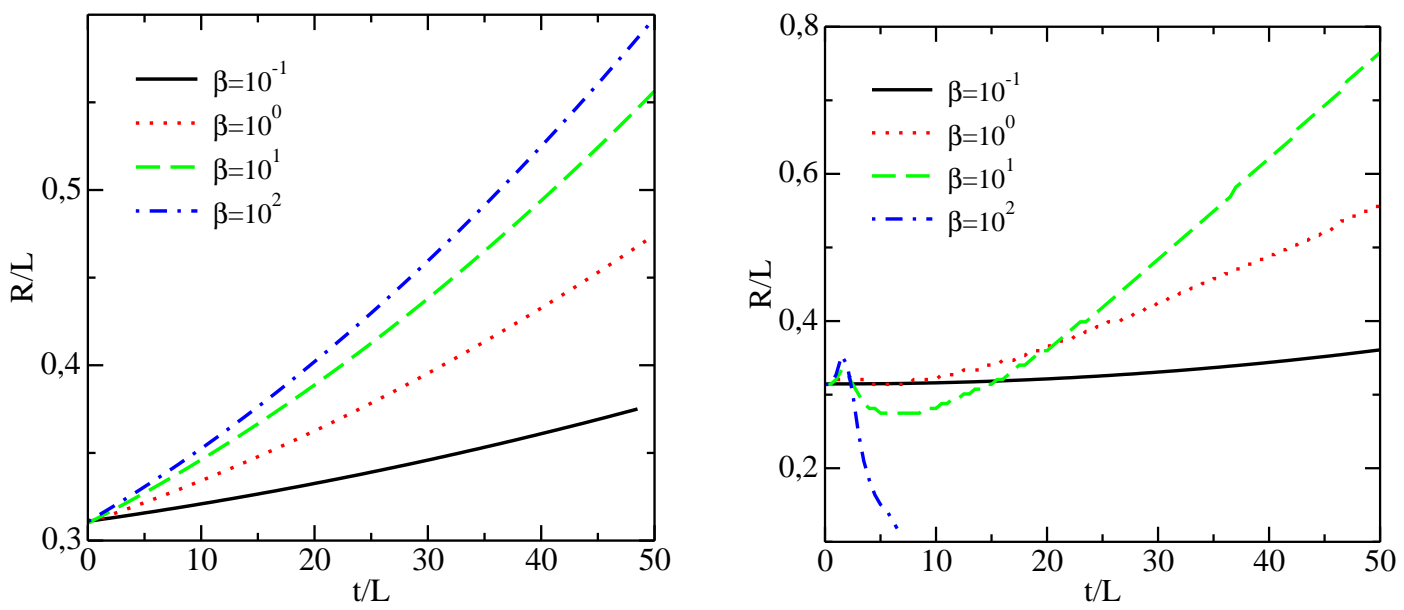

Fig. 6. Solution $R(t)$ for the liquid disk boundary, $\rho(R(t) ; t)=\rho_{c r}$, for several values of the viscosity. In left panel all parameters except viscosity are taken the same as in Fig. $4(\delta \mathcal{T}=0.02, L=30)$ and in right panel, as in Fig. $5(\delta \mathcal{T}=0.15, L=5)$.

of the growth of the seed (slop of the curve) increases with increase of $\beta$. Large values of the dimensional time presented in Fig. 6, $t \sim 50 L$, correspond to the value of the dimensionless time $\tau \sim 5 \sqrt{\beta}$. The latter value corresponds to the transition regime in the right panel of Fig. 3 (for all values of $\beta$ presented in Fig. 6). In this regime the velocity of the seed surface follows linear law. The asymptotic regime is achieved at much larger values of time. In the right panel of Fig. 6 results are presented for $T / T_{c r}=0.85$. For $t \gg t_{\text {rec }}$ the behavior is similar to that in the left panel. However at smaller values of time $\left(t \lesssim t_{\text {rec }}\right)$ for large values of $\beta$ (effectively small viscosity) there arise peculiarities. These peculiarities are associated with reconstruction of the initial density profile (87), occurring at $t \lesssim t_{\text {rec }}$. During this reconstruction period the typical radius of the seed may decrease. The dash curve demonstrates the same behavior, as is seen from the density profiles for time snapshots $2 L, 10 L$ and $20 L$ in the middle column of the upper panel of Fig. 5. If during the reconstruction process the size of the initially overcritical seed becomes smaller than the critical size, it causes subsequent shrinking of the seed. Namely this case is shown by the dash-dotted curve in the right panel of Fig. 6 . To check this statement we increased initial size of the seed at given $\beta$ and the behavior became similar to that shown by the dash curve. The larger $\beta$ and $|\delta \mathcal{T}|$, the higher is the seed velocity, cf. Eq. (34). For fixed $\beta$, evolution of the seed shown in the left panel $\left(T / T_{c r}=0.98, L=30 \mathrm{fm}\right)$ is slower than that demonstrated in the right panel $\left(T / T_{c r}=0.85, L=5 \mathrm{fm}\right)$. Thus peculiarities of the case of effectively low viscosity $(\beta \gg 1)$, which are seen in the right panel of the figure, are due to a larger inertia than in cases presented in the left panel.

In order to show how much the seed dynamics is sensitive to the choice of parameters of the EoS we take an another parameter choice $T_{c r}=162 \mathrm{MeV}$, $n / n_{\text {sat }}=1.3$, relevant for the hadron-sQGP phase transition. Results are presented in Fig. 7 for configurations with approximately the same $(P-$ 

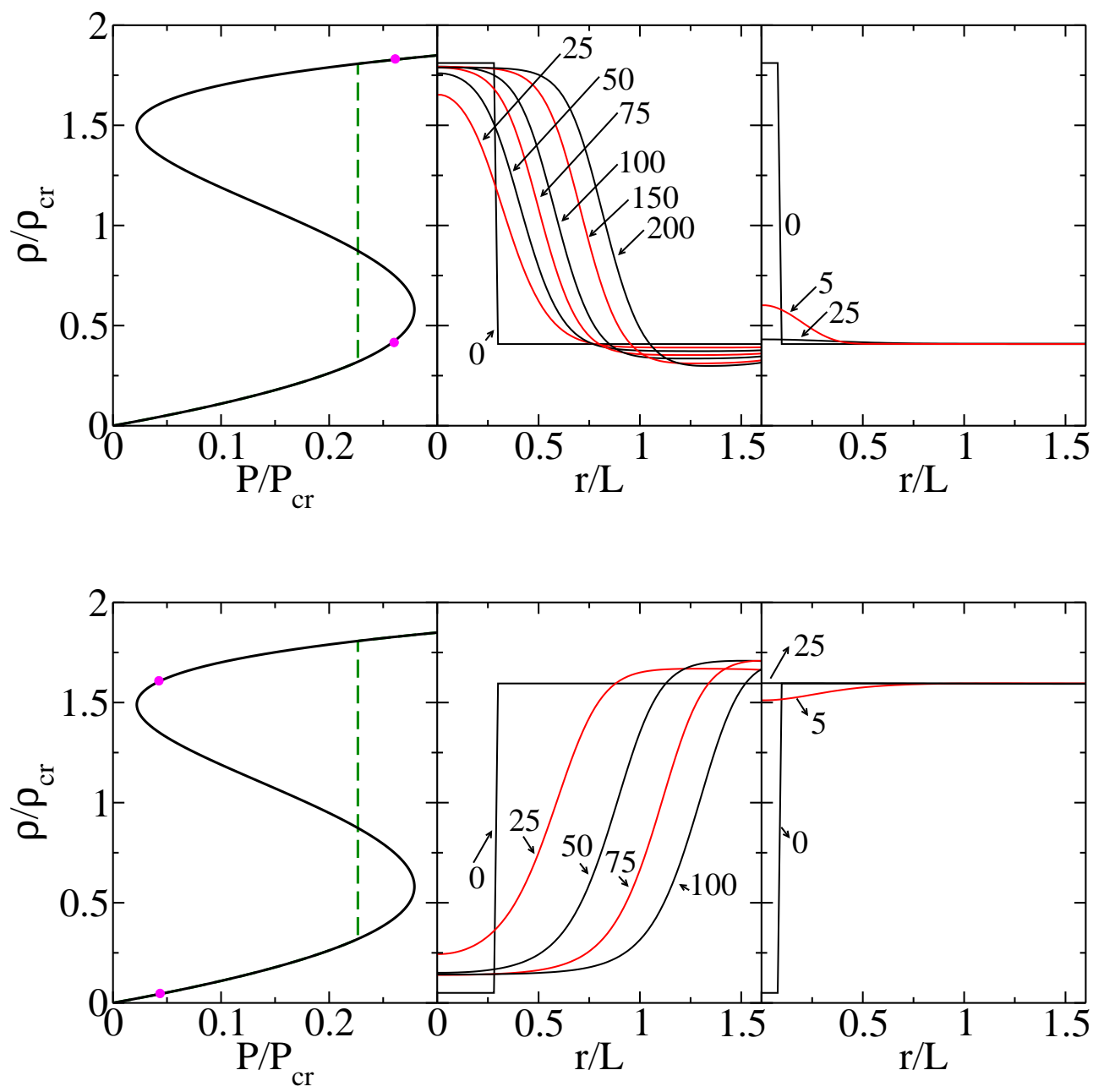

Fig. 7. The same as in Fig. 5 but for $T_{c r}=162 \mathrm{MeV}, n / n_{\text {sat }}=1.3, \eta \simeq 45 \mathrm{MeV} / \mathrm{fm}^{2}$ and for $\beta=0.2$.

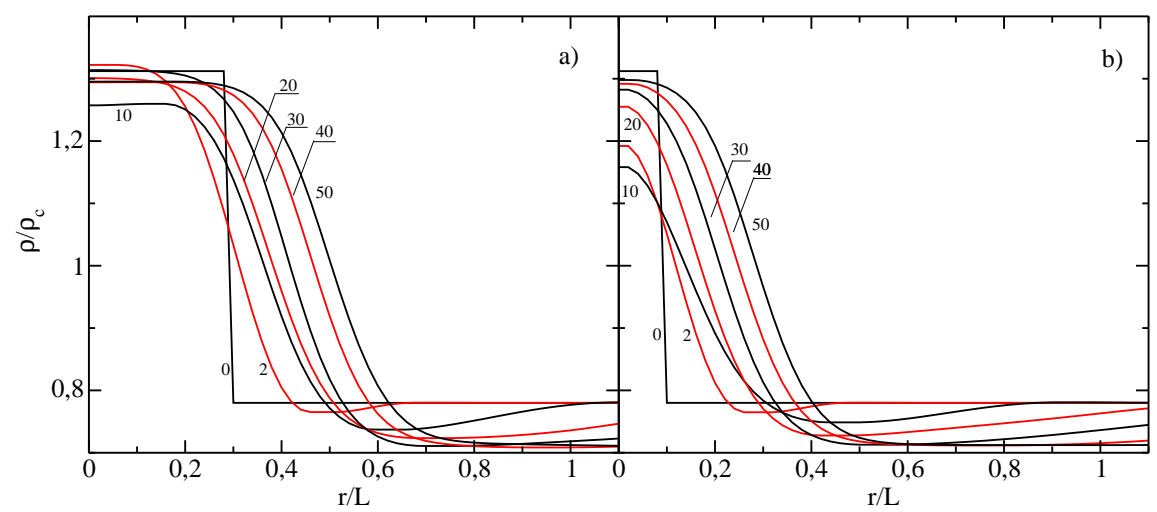

Fig. 8. Evolution of bands $\left(d_{\text {sol }}=1\right)$ of initially large (left) and small (right) sizes, $r=|x|$. Parameters are taken the same as in Fig. 5. 


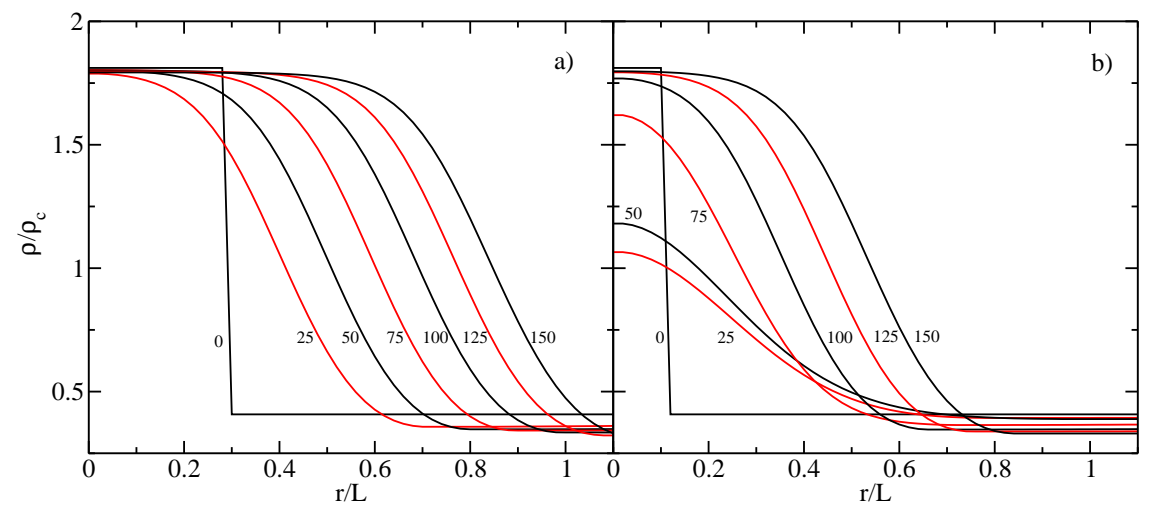

Fig. 9. The same as in Fig. 8 but for $|\delta \mathcal{T}|=0.15, L=5 \mathrm{fm}, \eta \simeq 23 \mathrm{MeV} / \mathrm{fm}^{2}$ and $\beta \simeq 0.042$.

$\left.P_{\min }\right) /\left(P_{\max }-P_{\min }\right)$ as shown in previous figures. As in Fig. 5 we take $T / T_{c r}=$ 0.85 and compute the configuration for $\eta \simeq 45 \mathrm{MeV} / \mathrm{fm}^{2}$ and for $\beta=0.2$ (effectively large viscosity). As we see, typical time scales and the shapes of configurations look similar to the case presented in Fig. 5, in spite of the parameter sets are completely different.

\subsection{Evolution of bands $\left(d_{\mathrm{sol}}=1\right)$ in $d=2$ spatial dimensions}

In Fig. 8 we show dynamics of liquid bands in metastable gas phase. Values $|\delta \mathcal{T}|=0.02, L=30 \mathrm{fm}$, are taken the same as in Fig. 5 and we again choose $T_{c r}=18.6 \mathrm{MeV}, n_{c r} / n_{\text {sat }}=0.42, \eta \simeq 3.2 \mathrm{MeV} / \mathrm{fm}^{2}$ and $\beta \simeq 12.6$ (effectively small viscosity). These solutions are similar to slabs in $d=3$. Left panel shows time evolution of a band of a large initial size $\left(R_{0}=0.3 L\right)$, whereas right panel demonstrates evolution of a band having initially rather small size $\left(R_{0}=0.1 L\right)$. In difference with disks (solutions with $d_{\text {sol }} \neq 2$ ) in both cases (for large and small initial sizes of bands) dynamics looks similar: bands of the stable phase, being prepared in the metastable phase, undergo growth to the new phase. Nevertheless, we also see that during the shape reconstruction the slab first begins to dissolve and then grows. This peculiarity appeared since initial form of the density distribution that we exploit in numerical calculations deviates from the form given by analytical solution (24). Thus even for slabs there might exist a small critical size, that depends on peculiarities of the initial density profile. Slabs having sizes smaller than this critical size could then completely dissolve.

Fig. 9 shows the same as Fig. 8, but for $|\delta \mathcal{T}|=0.15, L=5 \mathrm{fm}, \eta \simeq$ $23 \mathrm{MeV} / \mathrm{fm}^{2}$ and $\beta \simeq 0.042$ (effectively large viscosity). We see that the qualitative picture of the time evolution remains the same.

The probability to prepare a band in a fluctuation is tiny. However, bands 

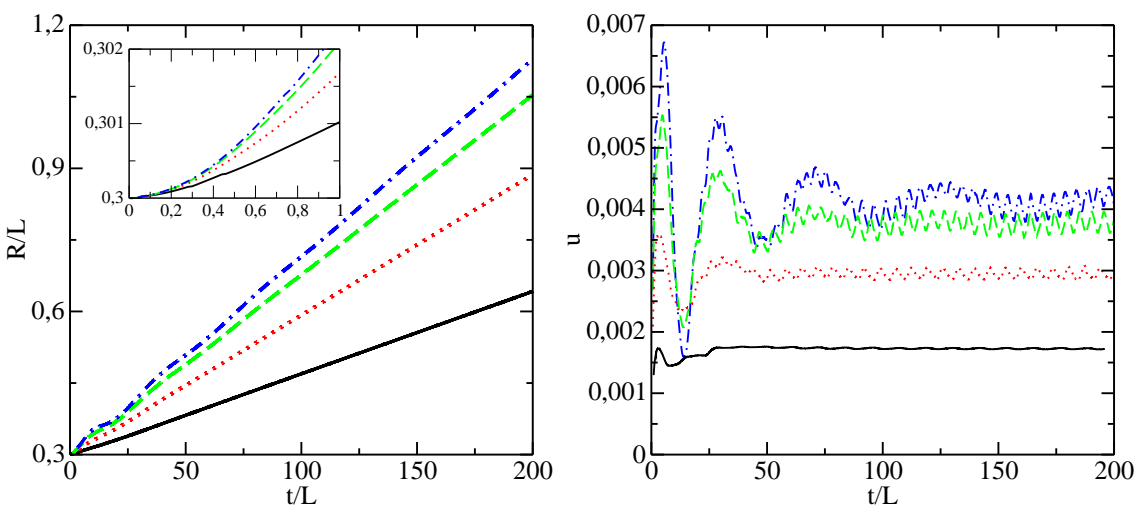

Fig. 10. Solution $R(t)$ (left panel) and $u=d R / d t$ (right panel) for the band boundary for several values of the viscosity. Values of $\beta$ and other parameters are taken the same as in left panel of Fig. 6 .

of the stable phase could be formed near the system boundary, provided the latter is flat.

In Fig. 10 we demonstrate the law for the growing with time of the band boundary $R(t)$ (in the left panel) and the velocity of the boundary $u=d R / d t$ (in the right panel) for different values of the viscosity. As for discs, the band boundary is specified as the point, where the density achieves the critical value $\left(\rho=\rho_{c r}\right)$. Results are presented for $T / T_{c r}=0.98, L=30 \mathrm{fm}$. For small values of time (see Figure insertion) $R(t)$ obeys the quadratic law, as it follows from Eq. (32). Solid curve (effectively large viscosity, $\beta=0.1$ ) follows the law (32) for $t>t_{\mathrm{rec}} \sim 100 \mathrm{fm}$. The higher $\beta$, the larger is deviation from this law since the reconstruction time increases then as $t_{\mathrm{rec}} \propto \sqrt{\beta}$. It is clearly demonstrated in the right panel, where we present the time dependence of the velocity of the seed growth. As follows from the Figure, even for large times the velocity $u$ does not obey the scaling law, $u \propto \sqrt{\beta}$ (as it would follow from Eq. (33)). This is so, because values of time $t \sim 200 L$ still correspond to the transition regime in the right panel of Fig. 3 (for all values of $\beta$ presented in Fig. 10). In this regime the velocity of the seed surface still slowly increases with time. The asymptotic regime is achieved at larger values of time (or for smaller $\beta$ at values of time shown in Figure).

Another important issue is presence of the damped long-wave oscillations which are clearly seen for all values of the effective viscosity. They occur at $t \lesssim t_{\text {rec }}$. Besides, in case of effectively small viscosity short-wave oscillations are clearly seen. As follows from Eq. (83), stable phase is, indeed, covered by fine ripples. 


\begin{tabular}{|c|c|c|}
\hline Viscosity/Wave number & Small & Large \\
\hline$k>k_{c r}$ & oscillation & damping \\
\hline$k<k_{c r}$ & growth & growth \\
\hline
\end{tabular}

Table 1

Different scenarios of the evolution of initial disturbances in spinodal region.

\subsection{Evolution of fluctuations in spinodal region}

As the initial density profile, we take now a wave developing on the background of a density $\bar{\rho}$ ( the latter value is chosen somewhere in the spinodal region), cf. sect. 2. The time dependent solution has the form

$$
\rho(t)=\bar{\rho}+A_{0} f(t) \sin (\vec{k} \vec{r})
$$

where $A_{0}$ is a small constant and $f(0)=1$.

We checked that in case of an effectively low viscosity $(\beta \gg 1)$ for $k>k_{c r}=$ $\sqrt{2}$ there appear oscillating modes and at an effectively high viscosity $(\beta \ll 1)$ there are only damped modes. For $k<k_{c r}=\sqrt{2}$ there are growing modes. Various regimes, as corresponding to growing, oscillating and damping initial disturbances, are indicated in Table 1.

In Fig. 11 we show time evolution of wave amplitudes given by Eq. (88) for an undercritical value of the wave number $k$ (left panel) and for an overcritical value (right panel). We take $T_{c r}=18.6 \mathrm{MeV}, n_{c r} / n_{\text {sat }}=0.42, c \simeq 5.56 \cdot 10^{-3}$ $\mathrm{fm}^{2}$. In case of the overcritical value $k$ and an effectively small viscosity $(\beta=$ 10) we demonstrate the change of the amplitude in the half-period of the oscillation. Such a behavior fully agrees with that follows from our analytical treatment of the problem, see (72), (82), (83). We have checked that in case of a small overcriticality (for $|\delta \mathcal{T}|=0.02$ ) slopes of the curves coincide up to the third digit with values of $\gamma$ calculated in subsect. 2.5. Even in case of sufficiently large deviation from the critical point (for $|\delta \mathcal{T}|=0.15$, as presented in Fig. 11), the difference of the curves $f(t)$ obtained numerically from those calculated analytically is less than $30 \%$.

In Fig. 12 we show time evolution of the wave amplitudes given by (88), for an undercritical value of the wave number $k$ (left panel) and for an overcritical value (right panel) for the parameter choice $T_{c r}=162 \mathrm{MeV}, n / n_{\text {sat }}=1.3$, as for the hadron-sQGP phase transition.

We see that in case relevant for the hadron-quark phase transition the evolution is more rapid compared to the example of the configuration presented in Fig. 11 relevant for the NGL phase transition. Nevertheless even in the former 

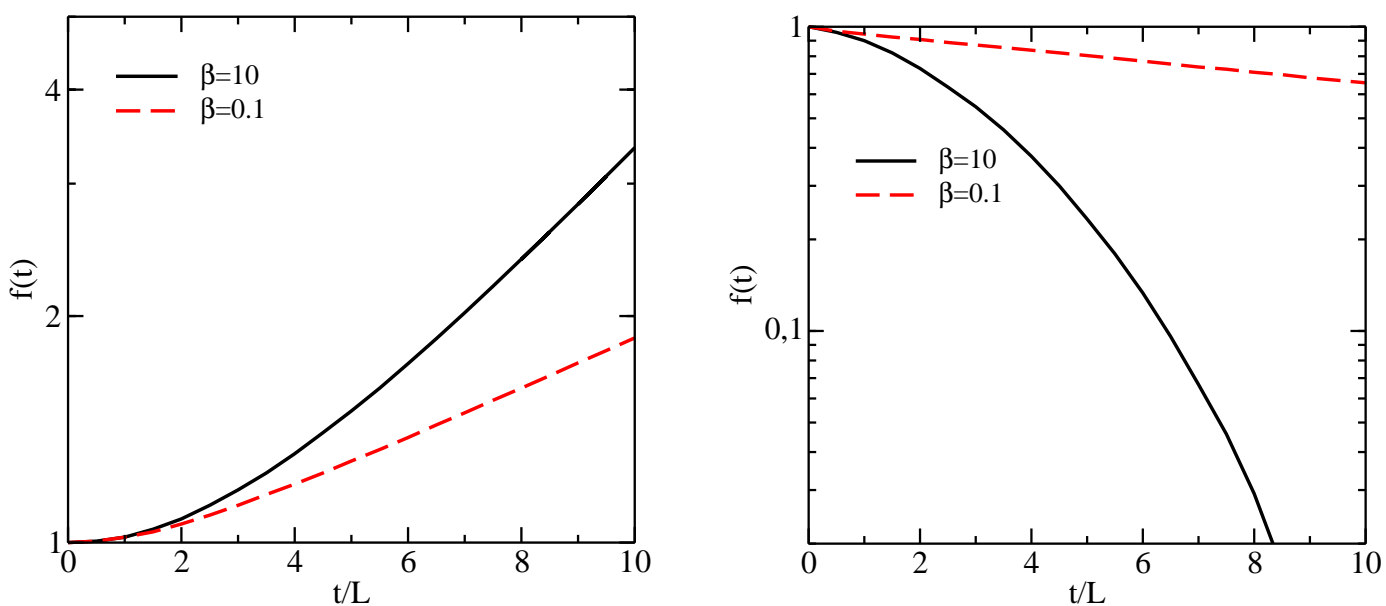

Fig. 11. Time evolution of the wave amplitudes $f(t)$, see Eq. (88), normalized to the amplitude of the initial disturbance. Solid line is for effectively small viscosity $(\beta=10)$ and dash line, for the large viscosity $(\beta=0.1)$. Left panel: the undercritical wave number $k=2 l / L$ (growing modes). Right panel: the overcritical value $k=8 l / L$ (oscillation modes for large $\beta$ and damped modes for small $\beta$ ). Other parameters are taken the same, as in Fig 5.
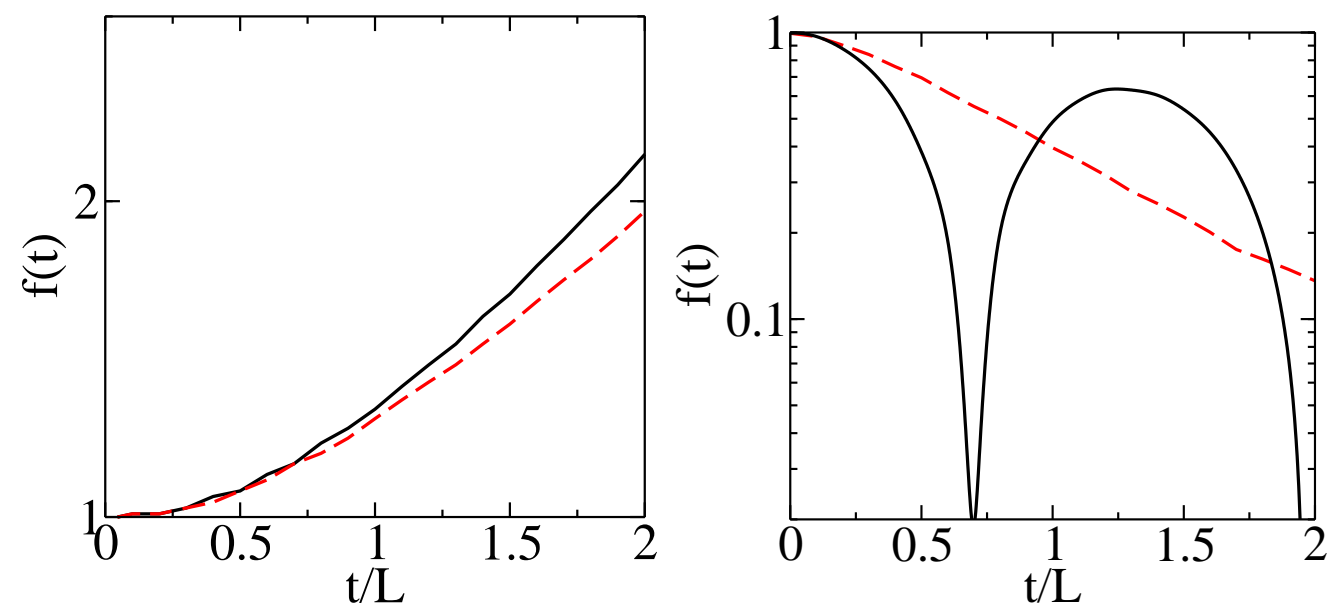

Fig. 12. The same as in Fig. 11 but for $T_{c r}=162 \mathrm{MeV}, n / n_{\text {sat }}=1.3$.

case the time evolution remains sufficiently slow, especially at an effectively large viscosity. Then the typical time scale $t \sim 10 \mathrm{fm}$ is comparable with the total time of the fireball expansion. Thus in heavy-ion collisions during expansion of the fireball the system may linger in the old phase for a while even at $T<T_{c r}$. This means that the equilibrium value of the critical temperature of the phase transition might be significantly higher than the value which may 


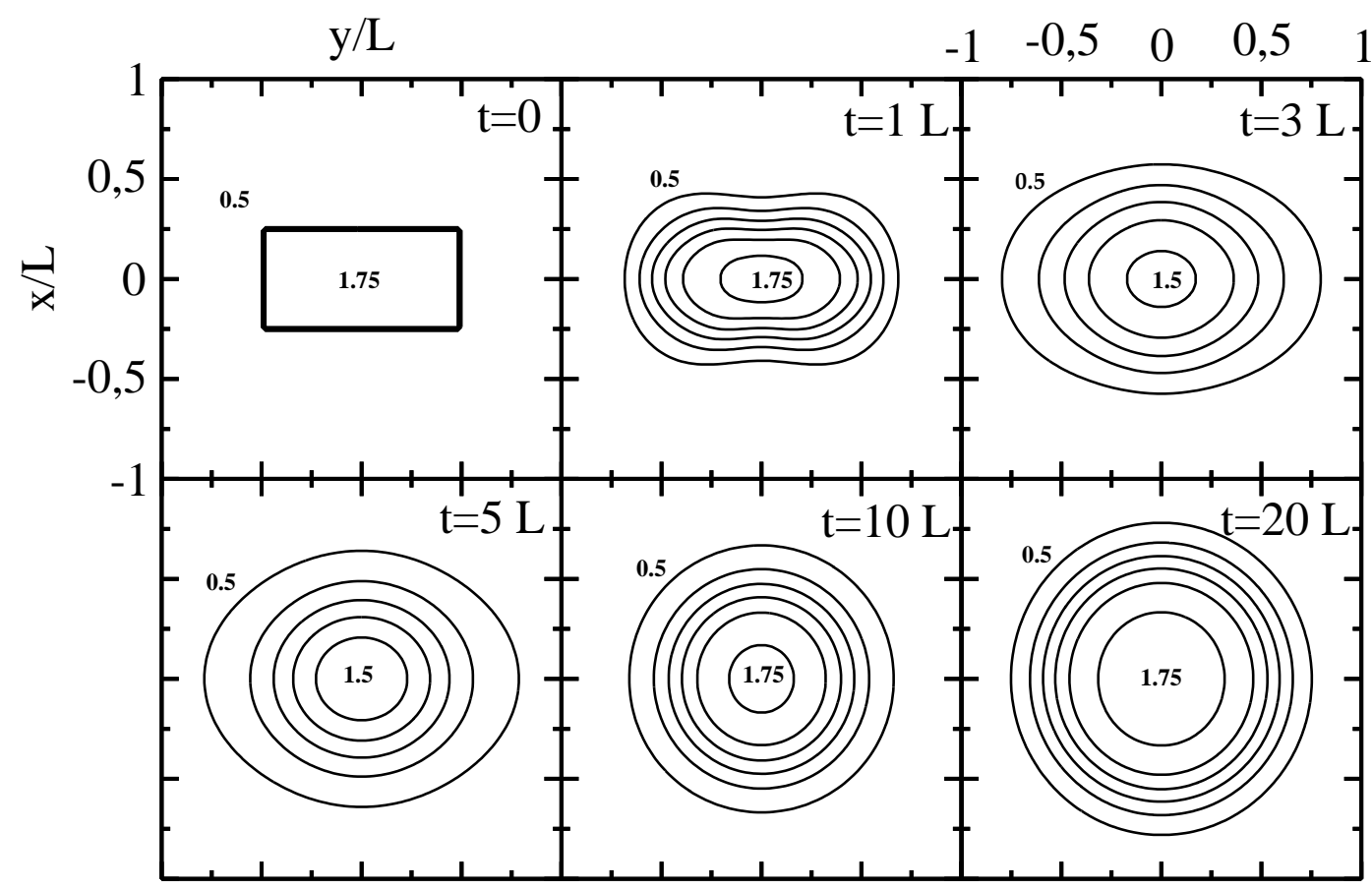

Fig. 13. Isolines of the density $n / n_{c r}$ demonstrating the time evolution of initially asymmetric disk of overcritical size (in both directions) for effectively large viscosity $(\beta=0.1)$. Density isolines are drawn with an increment equal 0.25 . Time snapshots are indicated in the sub-figures, $|\delta \mathcal{T}|=0.15, L=5 \mathrm{fm}$.

manifest in the growth of fluctuations in experiments.

\subsection{Evolution of asymmetric spots $\left(d_{\mathrm{sol}}=2\right)$ in $d=2$ space}

Now let us demonstrate how seeds having shapes significantly different from discs become the discs with passage of time. In Figs. 13 and 14 we show time evolution of the initially rectangular seed for effectively large $(\beta=0.1)$ and very small $\left(\beta=10^{3}\right)$ values of the viscosity, respectively. In case of an effectively large viscosity the shape of the seed monotonously transforms to the spherical one for typical time $t_{\text {rec }} \sim 30 \mathrm{fm}$. For an effectively small viscosity the dynamics is a more peculiar. In a line with our findings, see Eq. (62), one can recognize oscillations of the form. In the process of oscillations some pieces of matter first decouple with the growing seed and then fly away. Besides, the process lasts longer than in case of a large viscosity, $t_{\mathrm{rec}} \sim 150 \mathrm{fm}$. 


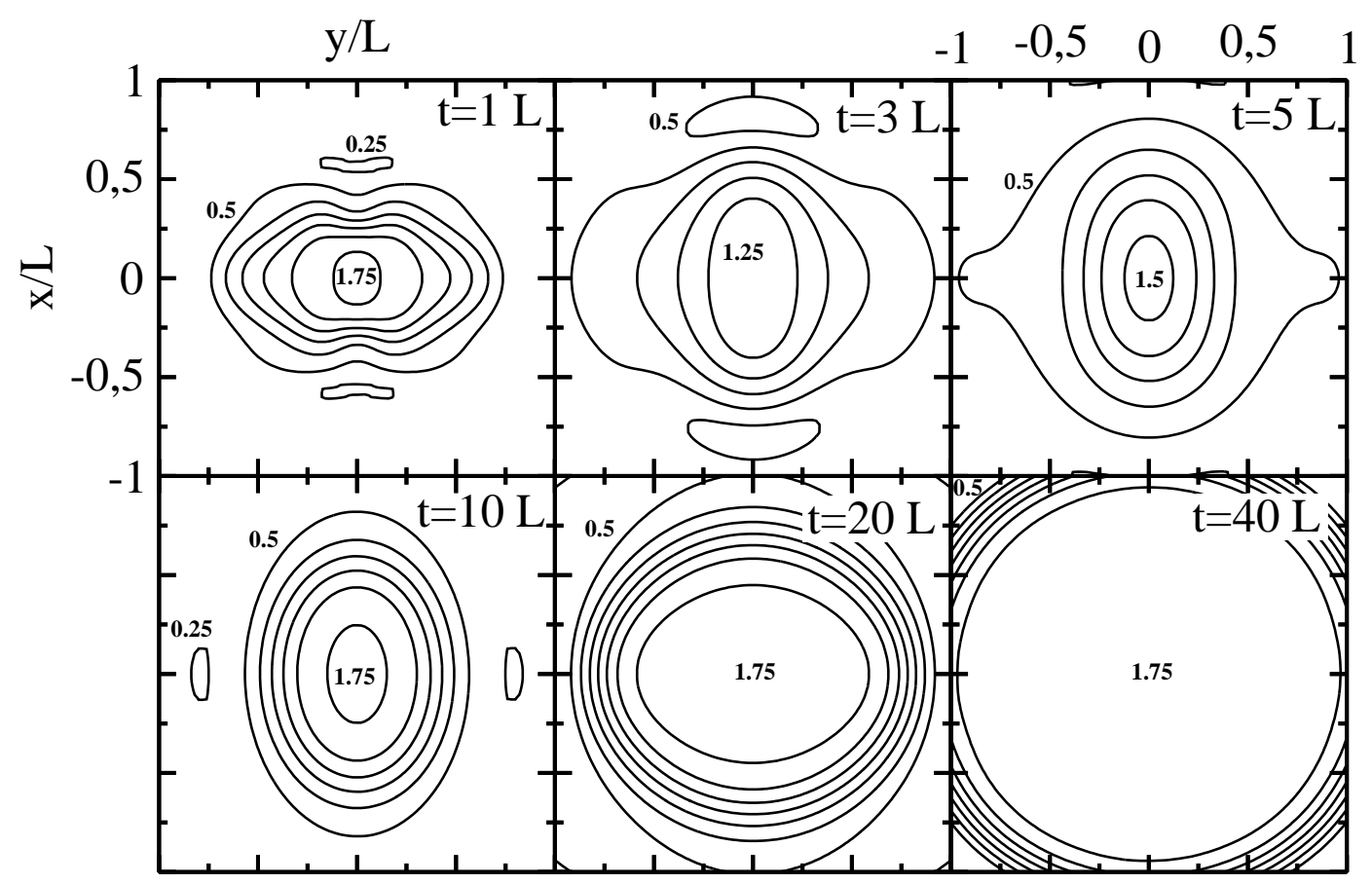

Fig. 14. The same as in Fig. 13, but for effectively very small viscosity $\beta=10^{3}$.

\section{The NGL and hadron - sQGP first-order phase transitions}

\subsection{The NGL phase transition}

For the description of the NGL first-order phase transition we take values $T_{c r} \simeq 18.6 \mathrm{MeV}, n_{c r} / n_{\text {sat }} \simeq 0.42, n_{\text {sat }} \simeq 0.16 \mathrm{fm}^{-3}$, similar to those one uses in the RMF models describing this transition, e.g. see [33]. We use the mVW model for the EoS, see Appendix A. Parameters of the EoS are then as follows: $a \simeq 3.14 \cdot 10^{2} \mathrm{MeV} \cdot \mathrm{fm}^{3}, b \simeq 5 \mathrm{fm}^{3}, \lambda \simeq 2.85 \cdot 10^{-6} \mathrm{fm}^{6} / \mathrm{MeV}^{2}$, $v^{2} \simeq 1.56 \cdot 10^{4}|\delta \mathcal{T}| \mathrm{MeV}^{2} / \mathrm{fm}^{6}, \epsilon^{\max } \simeq 2.16(\delta \mathcal{T})^{3 / 2} \mathrm{MeV} / \mathrm{fm}^{3}$.

In order to estimate the gradient coefficient " $c$ " we suppose that $l(T=0)=$ $d_{\text {dif }} \simeq 0.5 \mathrm{fm}$, see Eq. (19), where $d_{\text {dif }}$ is the diffusion length, as it follows from the Woods-Saxon parameterization of the density profile of the nucleus. Then $c \simeq 5.56 \cdot 10^{-3} \mathrm{fm}^{2}$, and for an appropriate value $|\delta \mathcal{T}| \simeq 0.15$ we find $l \simeq 1.3 \mathrm{fm}$. In order to obtain $l(T=0) \simeq 0.5 \mathrm{fm}$ we should take $\sigma_{0} \simeq 4 \mathrm{MeV} / \mathrm{fm}^{2}$. Then for $T=0.85 T_{c r}$ we get $\sigma \simeq \frac{1}{4} \mathrm{MeV} / \mathrm{fm}^{2}$. Using these values and taking $\eta \simeq 23 \mathrm{MeV} / \mathrm{fm}^{2}$ following Ref. [18], we obtain $\beta_{\mathrm{NGL}} \simeq 0.023$ that corresponds to the limit of effectively very large viscosity.

Then we evaluate the time scale $t_{0} \simeq 20|\delta \mathcal{T}|^{-1} \mathrm{fm}$, see Eq. (19). Also we are able to estimate values $t_{\rho}, t_{\text {dis }}$ as they follow from Eqs. (48), (57). We find $t_{\rho}\left(\gamma \epsilon^{\max }\right) \simeq 40 R \gamma^{-1}|\delta \mathcal{T}|^{-1 / 2}, t_{\rho}\left(R_{c r}, \gamma \epsilon^{\max }\right) \simeq 30 \gamma^{-2}|\delta \mathcal{T}|^{-1}, t_{\mathrm{dis}} \simeq 20 R(R / \mathrm{fm})$. 
The initial stage of the process occurring for $t \lesssim t_{\text {init }} \simeq 0.06|\delta \mathcal{T}|^{-1}$, see (36), proves to be very short $\left(t_{\text {init }} \ll t_{\rho}\right)$.

Further following [18] we evaluate thermal conductivity $\kappa \simeq 0.08 \mathrm{fm}^{-2}$ and estimate typical time for the thermal transport $t_{T}$. From (15) we find $t_{T} \simeq$ $3 R(R / \mathrm{fm})$. Here we used that for symmetric nuclear matter $c_{V} \simeq 3 n$ for $T \gg \epsilon_{\mathrm{F}}$ and $c_{V} \simeq 2(\pi / 3)^{2 / 3} m n^{1 / 3} T$ for $T \ll \epsilon_{\mathrm{F}}$. Thus the heat transport might become operative for $R>R_{\text {fog }}\left(\gamma \epsilon^{m a}\right) \simeq 13 \gamma^{-1}|\delta \mathcal{T}|^{-1 / 2} \mathrm{fm}$, where $R_{\text {fog }}$ is a typical radius of seeds in the "nuclear fog". Using that $R_{c r}\left(\gamma \epsilon^{\max }\right) \simeq$ $0.9 \gamma^{-1}|\delta \mathcal{T}|^{-1 / 2} \mathrm{fm}$, see Eq. (30), we obtain $R_{\text {fog }} \gg R_{c r}$. Thereby there is a long time interval, where the heat transport is not yet efficient and solutions presented in this paper are valid.

Assuming that the fireball reaches spinodal region, following (73) we may estimate the time scale for the formation of the aerosol, $t_{\mathrm{aer}}^{\eta} \sim 10|\delta \mathcal{T}|^{-1} \mathrm{fm}$, and the size scale for seeds in aerosol, $R_{\text {aer }}^{\eta} \simeq 2^{-1} \beta^{-1 / 4} l \simeq 0.6|\delta \mathcal{T}|^{-1 / 2} \mathrm{fm}$. With the help of Eq. (85) for $\beta \simeq 0.023$, we evaluate typical Reynolds numbers $\left.\operatorname{Re} \sim(0.1 \div 1) \ll \operatorname{Re}_{c r}\right)$, for $\alpha \sim 1 \div 10$.

We see that all relevant time scales for the formation of seeds are long ( $\gtrsim 10 \mathrm{fm})$ increasing, when the system comes closer to the critical point. Thus it is likely that only an initial and might be an intermediate stage of the NGL first-order phase transition may manifest itself in the course of heavy-ion collisions. Also it seems unlikely to observe effects of a narrow vicinity of the critical point of the phase transition, since the fireball is located in this region a short time compared with the time scale characterizing the growth of seeds. This also means that the critical temperature of the first-order phase transition calculated at assumption of the thermal equilibrium might be significantly higher than the value, which may manifest in the growth of fluctuations in experiments. Note that values of the critical temperature calculated in different models are in the range $T_{c r} \sim 15 \div 20 \mathrm{MeV}$, whereas experimental value, as it follows from the analysis of the multi-fragmentation is $T_{c r} \sim 5 \div 8 \mathrm{MeV}$, see [36]. One usually associates this difference with the finite size effects. We point out that at least partially the difference can be explained by the dynamical effects.

\subsection{The hadron-sQGP phase transition}

In case of the hadron-sQGP first-order phase transition critical values $T_{c r}$ and $n_{c r}$ are rather unknown and can be varied in a broad range. Values $T_{c r}=(80 \div 170) \mathrm{MeV}$ and $n_{c r}=(1 \div 6) n_{s a t}$ are used in different models. For rough estimates we take values $T_{c r} \simeq 162 \mathrm{MeV}, n_{c r} / n_{\text {sat }} \simeq 1.3$, as they are obtained following lattice calculations, cf. [27]. We again use the $\mathrm{mVW}$ model for the EoS, see Appendix A. Parameters of the EoS are then as fol- 
lows: $a \simeq 8.76 \cdot 10^{2} \mathrm{MeV} \cdot \mathrm{fm}^{3}, b \simeq 1.60 \mathrm{fm}^{3}, \lambda \simeq 7.80 \cdot 10^{-5} q^{-3} \mathrm{fm}^{6} / \mathrm{MeV}^{2}$, $v^{2} \simeq 1.56 \cdot 10^{4} q^{2}|\delta \mathcal{T}| \mathrm{MeV}^{2} / \mathrm{fm}^{6}, \epsilon^{\max } \simeq 58.4 \cdot|\delta \mathcal{T}|^{3 / 2} \mathrm{MeV} / \mathrm{fm}^{3}, m_{q}$ is the effective quark mass, $q=m_{q} /(300 \mathrm{MeV})$. Further, we obtain $l(T=0) \simeq 0.2 \mathrm{fm}$ (radius of confinement) for $\sigma_{0} \simeq 40 \mathrm{MeV} / \mathrm{fm}^{2}$. If one used $\sigma_{0} \simeq 100 \mathrm{MeV} / \mathrm{fm}^{2}$, one would estimate $l(T=0) \simeq 0.5 \mathrm{fm}$.

Next we estimate $s(T) \simeq 7 T^{3}\left(T / T_{c r}\right), c_{V} \simeq 28 T^{3}\left(T / T_{c r}\right)$ at $T$ near $T_{c r}$, as it follows from the lattice data [49]. Assuming minimal value of the viscosity $\eta_{\min }=s /(4 \pi)=60 \mathrm{MeV} / \mathrm{fm}^{2}, \zeta_{\min }=0$ we evaluate maximum value of $\beta$ : $\beta_{\mathrm{sQGP}}^{\max } \simeq 0.015 q$ for $\sigma_{0} \simeq 40 \mathrm{MeV} / \mathrm{fm}^{2}[50]$, that corresponds to the limit of effectively very large viscosity. Even for $\sigma_{0} \simeq 100 \mathrm{MeV} / \mathrm{fm}^{2}, m_{q}=600 \mathrm{MeV}$ we would get $\beta_{\mathrm{sQGP}}^{\max } \simeq 0.2 \ll 1$. Note that following $[20]$ the bulk viscosity diverges in the critical point. If were so $(\beta \rightarrow 0)$, the quark-hadron system would behave as absolutely viscous fluid, like glass, in near critical region. Contrary, Refs. [21,12] argue for a smooth behavior of the bulk viscosity.

With $\beta=0.015$, we further estimate $t_{0} \simeq 2|\delta \mathcal{T}|^{-1} \mathrm{fm}, t_{\rho}\left(\gamma \epsilon^{\max }\right) \simeq 9.1 R \gamma^{-1} q^{1 / 2}|\delta \mathcal{T}|^{-1 / 2}$ and $t_{\text {dis }} \simeq 14 q\left(R_{0} / \mathrm{fm}\right) R_{0}$. The time scale for the formation of the aerosol is $t_{\mathrm{aer}}^{\eta} \simeq|\delta \mathcal{T}|^{-1} \mathrm{fm}$, and the size scale for seeds in aerosol is $R_{\mathrm{aer}}^{\eta} \simeq 0.24|\delta \mathcal{T}|^{-1 / 2} \mathrm{fm}$. Only $t_{\text {init }}^{\eta} \simeq 0.03 q|\delta \mathcal{T}|^{-1}$ fm proves to be small (excluding quite small $\delta \mathcal{T}$ ).

For the thermal conductivity we use an estimation $\kappa \simeq \alpha_{0} \eta / m$, see [17]. Factor $\alpha_{0}$ depends on the EoS used. We will take $\alpha_{0}=3$. Then one recovers appropriate relation between values of $\kappa$ and $\eta$ for NGL transition, which we have used above, see [18]. For the hadron-sQGP transition this estimation renders $\kappa_{\mathrm{sQGP}} \simeq 3 \eta / m_{q}$. Then we are able to evaluate the scale of the heat transport time, $t_{T} \simeq 26 q(R / \mathrm{fm})^{2} \mathrm{fm}$. The heat transport becomes operative for $R>R_{\text {fog }}\left(\gamma \epsilon^{\max }\right) \simeq 0.3 \gamma^{-1} q^{-1 / 2}|\delta \mathcal{T}|^{-1 / 2}$ fm. Here $R_{\text {fog }}$ is the scale of size of the seed in the quark (or hadron) fog-like state. Using that $R_{c r}\left(\gamma \epsilon^{\max }\right) \simeq$ $0.3 \gamma^{-1}|\delta \mathcal{T}|^{-1 / 2}\left(\sigma_{0} /\left(40 \mathrm{MeV} / \mathrm{fm}^{2}\right)\right) \mathrm{fm}$, for $\sigma_{0}=(40 \div 100) \mathrm{MeV} / \mathrm{fm}^{2}$ we obtain $R_{\mathrm{fog}} \lesssim R_{c r}$. Thereby, the heat transport might be always operative for the description of the evolution of overcritical seeds in hadron-sQGP phase transition. The value $R_{\text {fog }}$ proved to be very small ( $\left.\lesssim 0.1 \div 1 \mathrm{fm}\right)$. However typical time $t_{T}$ is rather long. Therefore, the system most probably would have no time to fully develop a fog-like state in a hadron-quark phase transition in heavy-ion collisions.

For the system located in the vicinity of the critical point all estimated time scales (except $t_{\text {init }}$ ) are very large. If the system trajectory paths rather far from the critical point, all time scales, except $t_{T}$, become of the order or less than the typical life-time of the fireball ( $\sim 10 \mathrm{fm}$ at RHIC conditions). Reynolds numbers are $\lesssim 1$, being much smaller than $\operatorname{Re}_{c r} \gtrsim 1000$. Thereby, turbulence regime is not reached. 


\section{Conclusion}

In this paper we studied the dynamics of systems undergoing first-order phase transitions. We formulated analytical description of the problem (in general case in $d+1$-dimensional space-time) valid for systems in the vicinity of the critical point. The analytical solutions were derived for configurations of certain symmetries (droplets/bubbles, rods and slabs for $d=3$ ).

Then, the general system of equations of non-ideal non-relativistic hydrodynamics was numerically solved for a modified Van der Waals equation of state. Results for the original Van der Waals equation of state can be obtained by simple re-scaling of the time and the viscosity. Since there exist many different regimes for the relevant processes, in this paper we partially restricted our analysis. We did not incorporate the heat transport, which governs evolution of seeds of a large size. Also we focused on the case, when concentration of seeds of the new phase in the old phase is still rather small and one can ignore their coalescence. For simplicity we did not generate fluctuations in random processes considering evolution of the given seed after it has been produced in a fluctuation. Generalizations will be presented elsewhere. With mentioned reservations we revealed and studied general features of the dynamics of firstorder phase transitions. These main features are as follows:

(i) Essential role in the dynamics of the first-order phase transitions is played by the viscosity effects. This might be very important, since existing threedimensional hydrodynamical schemes pretending to study phase transitions exploit equations of ideal hydrodynamics, whereas viscosity effects are simulated only implicitly, e.g., with the help of phenomenological coefficients responsible for a friction of fluids.

(ii) Because of the surface tension (except for one-dimensional slabs) there exists a critical size for the seeds of the stable phase. Seeds of overcritical sizes grow with time, while undercritical fluctuations dissolve. The closer to the critical point, the slower are processes. Even far from the critical point overcritical seeds grow slowly.

(iii) We have shown that seeds with an asymmetric shape become spherical with time. This process is slow. For systems with effectively large viscosity the shape of the seed changes steadily to the spherical one. For systems with effectively small viscosity the seed undergoes long-wave and short-wave damped oscillations in the process of acquiring spherical quasi-equilibrium shape. Short-wave oscillations damp very slowly.

(iv) In the spinodal region the system is unstable against generation of waves (with not too high wave-number). However instability develops slowly, if the system is close to the critical point (the time scale tends to infinity at the critical point). Far from the critical point processes become more rapid. The latter observation can be very important for studying possible signatures of 
the gas-liquid and hadron-quark first-order phase transitions in heavy-ion collisions.

As a signal of the phase transition, some models suggest to search anomalies in the behavior of the derivatives of thermodynamic quantities, e.g. specific heat. These anomalies appear due to fluctuations, whereas we have shown that those anomalies in fluctuations may not have sufficient time to develop. Moreover we conclude that in heavy-ion collisions the system may linger in the old phase (e.g. in the QGP state, or in the gas state) longer during the fireball expansion, even when $T(t)$ has already decreased below the corresponding value of the equilibrium critical temperature of the phase transition. In another words, this means that the value of the critical temperature calculated within equilibrium thermodynamical models might be significantly higher than the value, which may manifest in the growth of fluctuations in experiments.

Typical dimensionless parameter $\beta$ that separates effectively viscous and perfect fluid regimes proves to be $\propto \sigma_{0}^{2} /\left(\frac{4}{3} \eta+\zeta\right)^{2}$, where $\sigma_{0}$ is the surface tension at $T=0$ and $\eta$ and $\zeta$ are the shear and bulk viscosities. According to our estimates the system undergoing nuclear gas-liquid phase transition in the course of heavy-ion collisions at low energies represents effectively very viscous fluid $(\beta \ll 1)$. There exist arguments that strongly coupled quark-gluon plasma state, which is, as commonly expected, formed in heavy-ion conditions at RHIC, represents almost perfect fluid in the cross-over region (see e.g. [11]). Estimating the ratio of the viscosity to the entropy density as $\eta / s<0.2$ it was concluded [12] that strongly coupled quark-gluon plasma is the most perfect liquid known. It is usually believed that this property of the plasma will survive at finite baryon density for systems in the vicinity of the critical point of the first-order phase transition (critical end point). In contrast our estimates show that the system undergoing the hadron-quark first-order phase transition in the course of violent heavy-ion collisions represents effectively very viscous fluid $(\beta \ll 1)$.

We found that the heat transport effects may play important role in description of the hadron-sQGP phase transition dynamics, whereas these effects are much less pronounced in the case of the nuclear gas-liquid transition.

In future we plan to use a realistic equation of state to study the heavy-ion collision dynamics.

After our paper has been submitted to the journal there appeared interesting paper [51] devoted to the description of fluctuations in the spinodal region at the hadron-sQGP first-order phase transition which well complements our 
study.

\section{Acknowledgements}

We are grateful to B. Friman, Y.B. Ivanov, E.E. Kolomeitsev, J. Randrup, and V.D. Toneev for numerous discussions and valuable remarks. Especially we are grateful to D. Blaschke and L. Grigorenko for the reading of the manuscript and making numerous useful comments. This work was supported by the Russian Foundation for Basic Research RFBR grant 08-02-01003-a and the $\mathrm{BMBF} / \mathrm{WTZ}$ project RUS 08/038.

\section{Appendix A. Modified Van der Waals EoS}

The best known example to illustrate principal features of the first-order phase transition is the Van der Waals fluid. The pressure is given by

$$
P_{\mathrm{VW}}[V, T]=\frac{N T}{V-N b}-\frac{N^{2} a}{V^{2}}=\frac{n T}{1-b n}-n^{2} a
$$

where parameter " $a$ " governs the strength of the mean field attraction and " $b$ " controls a short-range repulsion. Obviously realistic EoS of nuclear matter has much more complicated temperature dependence. Due to this we will exploit a mVW EoS with

$$
P[V, T]=f(T) P_{\mathrm{VW}}[V, T]
$$

where $f(T)$ is a function of the temperature. In the given paper we do not consider the heat transport, assuming $T=$ const. In this case our solutions are self-similar. Doing replacement $t \rightarrow t \sqrt{f(T)}$ and $\left(\tilde{d} \eta_{\mathrm{r}}+\zeta_{\mathrm{r}}\right) \rightarrow\left(\tilde{d} \eta_{\mathrm{r}}+\zeta_{\mathrm{r}}\right) / \sqrt{f(T)}$ we recover results for the original VW EoS.

We choose $\rho_{\mathrm{r}}=\rho_{c r}, T_{\mathrm{r}}=T_{c r}$ as the reference point (see notations in subsect. 2.1) and perform expansion of the pressure in the vicinity of this point. The critical liquid-gas point (evaporation point) is determined from the conditions $\partial P / \partial V=\partial^{2} P / \partial V^{2}=0, \partial^{3} P / \partial V^{3}<0$. Critical parameters are

$$
T_{c r}=\frac{8 a}{27 b}, \quad V_{c r}=3 N b, \quad P_{c r}=\frac{a}{27 b^{2}}, \quad n_{c r}=\frac{1}{3 b}
$$

For the given EoS they coincide with those for purely VW EoS. 
For $T=T_{\text {comp }}=\frac{27}{32} T_{c r} \simeq 0.844 T_{c r}, n_{\text {comp }}=\frac{3}{2} n_{c r}$ pressure (89) touches zero. For $T<T_{\text {comp }}$ there arises density interval (from $\left(\frac{3}{2}-\sqrt{\frac{9}{4}-\frac{8}{3} \frac{T}{T_{c r}}}\right) n_{c r}$ to $\left(\frac{3}{2}+\right.$ $\left.\sqrt{\frac{9}{4}-\frac{8}{3} \frac{T}{T_{c r}}}\right) n_{c r}$ ), where pressure becomes negative. In this paper we will restrict our consideration by taking $T_{c r}>T>T_{\text {comp }}$.

For a more convenient analytical treatment of the problem let us, selecting corresponding function $f(T)$ additionally fulfill condition $\left.\frac{\partial P}{\partial T}\right|_{\left(n_{c r}, T_{c r}\right)}=0$. It allows to parameterize the Helmholtz free energy, as it has been done in the paper body for the Landau free energy, with two minima deviating only little from each other. Then we may specify function $f$, e.g., as

$$
f(\delta \mathcal{T}) \simeq C(\delta \mathcal{T})[1-2 \delta \mathcal{T}]^{2}
$$

Although our analytical consideration is valid only in the vicinity of the critical point $0<-\delta \mathcal{T} \ll 1$ and we perform numerical calculations for $0<-\delta \mathcal{T}$, let us specify the pre-factor $C(\delta \mathcal{T})=\left[1+4(\delta \mathcal{T})^{2}\right]^{-1}$ to reproduce the ideal gas EoS for sufficiently low $n$ and high $T$.

Expanding the pressure in $|\delta \mathcal{T}| \ll 1, \delta n / n_{c r} \ll 1$ we get

$$
\delta P=\frac{9 T_{c r} \delta \mathcal{T} \delta n}{4}+\frac{9 T_{c r}}{16 n_{c r}^{2}}(\delta n)^{3}-6 T_{c r} n_{c r}(\delta \mathcal{T})^{2}+\ldots
$$

Last term in (93) is actually unimportant since addition of any constant (at $T=$ const last term is constant) does not change equations of motion. Let us count $P$ from its value in the final equilibrium state (reaching at $t \rightarrow \infty$ ). Namely this difference $P-P_{f}$ has the meaning of the thermodynamical force driving the system to the final equilibrium state (see (13)). Then we may construct the Landau free energy (16) such that $\delta\left(\delta F_{L}\right) / \delta(\delta n)=P-P_{f}$. Comparing (93) with (17) we find relations between coefficients:

$$
\begin{aligned}
a & =9 T_{c r} /\left(8 n_{c r}\right), \quad b=1 /\left(3 n_{c r}\right), v^{2}=-3 m^{2} T_{c r} \delta \mathcal{T} /(2 a b)=-4 \delta \mathcal{T} n_{c r}^{2} m^{2} \\
\lambda & =\frac{3 a b}{2 m^{3}}=\frac{9}{16} \frac{T_{c r}}{n_{c r}^{2} m^{3}}, \quad \epsilon=n_{c r}\left(\mu_{i n}-\mu_{f}\right) \\
t_{0} & =8\left(\widetilde{d} \eta_{\mathrm{r}}+\zeta_{\mathrm{r}}\right)\left(9 n_{c r} T_{c r}|\delta \mathcal{T}|\right)^{-1}
\end{aligned}
$$

Since we used $f\left(T \rightarrow T_{c r}\right)=1$ in Eq. (92), these relations are the same as for the original VW EoS. 


\section{Appendix B. Mean field and fluctuation region}

In the paper body we considered dynamics of fluctuations assuming that thermodynamical characteristics like pressure, free energy etc., are given (mean field approximation) and not modified by fluctuations (mean field approximation). In order to estimate a possible influence of fluctuations on thermodynamical characteristics of the equilibrium uniform system at $T \neq 0$ let us compare mean field and fluctuation contributions to the density of the specific heat $\delta c_{V}=-T\left[\partial^{2} \delta \mathcal{F} /(\partial T)^{2}\right]_{V}$. Using (22), (94) for $T$ near $T_{c r}$ we find

$$
c_{V}^{\mathrm{MF}} \simeq 9 n_{c r} / 2
$$

for configurations corresponding $|\epsilon| \ll \epsilon^{\max }$.

The fluctuation contribution to the specific heat density $c_{V}^{\prime}$ can be found with the help of the functional integration

$$
\exp \left[\delta F^{\prime} / T\right]=\int D \delta n^{\prime} \exp \left(\delta F^{\prime}\left[\delta n^{\prime}\right] / T\right)
$$

where following (16) we have

$$
\delta F^{\prime}=\frac{T}{2} \int \frac{d^{3} k}{(2 \pi)^{3}} \ln \left[\vec{k}^{2}+\alpha\right], \quad \alpha=2 \lambda v^{2} / c .
$$

From here using (28), (94) we obtain

$$
c_{V}^{\prime}=\frac{\alpha^{3 / 2}}{16 \pi|\delta \mathcal{T}|^{1 / 2}}=\frac{108}{\pi} \frac{n_{c r}^{3} T_{c r}^{3}}{\sigma_{0}^{3} \mid \delta \mathcal{T}^{1 / 2}} .
$$

Equating (95) and (98) (Ginzburg - Levanyuk criterion) we estimate the Ginzburg number

$$
\mathrm{Gi}=\frac{T_{c r}-T_{\mathrm{fl}}}{T_{c r}}=\left(\frac{24}{\pi} \frac{n_{c r}^{2} T_{c r}^{3}}{\sigma_{0}^{3}}\right)^{2} .
$$

Fluctuation region is narrow provided $\mathrm{Gi} \ll 1$ and it is broad for $\mathrm{Gi} \gtrsim 1$. Similar estimate to $\mathrm{Gi} \ll 1$ follows from the so-called Ginzburg criterion $\frac{4 \pi}{3} l^{3}\left|\delta \mathcal{F}^{\mathrm{MF}}\right| \gg T_{c r}$. In the fluctuation region $\left(T_{c r}>T>T_{\mathrm{fl}}\right)$ fluctuation effects may modify $\delta \mathcal{T}$ dependence of coefficients in Eq. (93).

Substituting in (99) typical values of parameters for the hadron-quark phase transition we estimate $\mathrm{Gi} \gtrsim 1.4\left(100 \mathrm{MeV} \cdot \mathrm{fm}^{-2} / \sigma_{0}\right)^{6}$, i.e. fluctuation region is 
broad. In case of the NGL phase transition we estimate $\mathrm{Gi} \sim 10\left(T_{c r} / 18.6 \mathrm{MeV}\right)^{6}$ and fluctuation region is also broad.

One can construct description of the fluctuation region incorporating effect of long-range fluctuations directly in the phenomenological expressions for the free energy $\delta F$ and the pressure $\delta P$. One can do it with the help of the replacements $v^{2} \rightarrow v_{\text {ren }}^{2}=v^{2}(T=0)|\delta \mathcal{T}|^{2 / 3}$, and $\lambda \rightarrow \lambda_{\text {ren }}=\lambda(T=0)|\delta \mathcal{T}|^{2 / 3}$ (similarly one incorporates fluctuations in description of the superfluid $\mathrm{He}^{4}$ ). Other coefficients in expressions of sub-section 2.2 remain unchanged. With these replacements dependence on $|\delta \mathcal{T}|$ disappears from the Ginzburg criterion, and both values $c_{V}^{\mathrm{MF}}$ and $c_{V}^{\prime}$ do not vanish for $\delta \mathcal{T} \rightarrow 0$. The Ginzburg

- Levanyuk criterion then reads as $\left(\frac{128}{3 \pi} \frac{n_{c r}^{2} T_{c r}^{3}}{\sigma_{0}^{3}}\right)^{2} \ll 1$. Effect of not included fluctuations is small provided this inequality is fulfilled.

Finally it is worthwhile to mention that it takes a long time (typically $\sim t_{0}$ ) to develop critical fluctuations. Therefore if the system passes the fluctuation region during a time $t_{\text {evol }}$, as it occurs in a course of heavy ion collisions, and $t_{\text {evol }}<t_{0}$, critical fluctuations will not have enough time to develop. Thus for $t<t_{0}$ it is legitimate to use mean field EoS to describe evolution of the system.

\section{References}

[1] E.M. Lifshiz, and L.P. Pitaevsky, "Physical Kinetics", Pergamon, 1981.

[2] A.Z. Patashinsky, and B.I. Shumilo, JETP 50, 712 (1979).

[3] A.B. Migdal, E.E. Saperstein, M.A. Troitsky, and D.N. Voskresensky, Phys. Rep. 192, 179 (1990).

[4] D.N. Voskresensky, Phys. Scripta 47, 333 (1993).

[5] A. Onuki, Phys. Rev. E7, 036304 (2007).

[6] H. Stoecker, and W. Greiner, Phys. Rep., 137, 277 (1986).

[7] Yu.B. Ivanov, V.N. Russkikh, and V.D. Toneev, Phys. Rev. C73, 044904 (2006).

[8] D. Teaney, J. Lauret, and E.V. Shuryak, Phys. Rev. Lett., 86, 4783 (2001); P.F. Kolb, U.W. Heinz, P. Huovinen, K.J. Eskola, and K. Tuominen, Nucl. Phys. A696, 197 (2001); T. Hirano, and K. Tsuda, Phys. Rev. C66, 054905 (2002); P. Kolb, and R. Rapp, Phys. Rev. C67, 044903 (2003).

[9] D. Teaney, Phys. Rev. C68, 034913 (2003).

[10] P. Romatschke, Eur. Phys. J. C52, 203 (2007)

[11] P. Romatschke, and U. Romatschke, Phys. Rev. Lett. 99, 172301 (2007). 
[12] E. Shuryak, Prog. Part. Nucl. Phys. 62, 48 (2009).

[13] A. Muronga, and D. H. Rischke, arXiv: nucl-th/0407114.

[14] Y. Lallouet, D. Davesne, and C. Pujol, Phys. Rev. C67, 057901 (2003).

[15] C. Nonaka, and M. Asakawa, Phys. Rev. C71, 044904 (2005); Nucl. Phys. A774, 753 (2006).

[16] K. Paech, H. Stoecker, and A. Dumitru, Phys. Rev. C68, 044907 (2003); K. Paech, and A. Dumitru, Phys. Lett. B623, 200 (2005); C.E. Aguiar, E.S. Fraga, and T. Kodama, J. Phys. G32, 179 (2006).

[17] V.M. Galitsky, Yu.B. Ivanov, and V.A. Khangulian, Sov. J. Nucl. Phys. 30, 401 (1979).

[18] L. Shi, and P. Danielewicz, Phys. Rev., C68, 064604 (2003).

[19] L.P. Csernai, J.I. Kapusta, and L.D. McLerran, Phys. Rev. Lett., 97, 152303 (2006).

[20] D. Kharzeev, and K. Tuchin, JHEP 0809, 093 (2008); F. Karsh, D. Kharzeev, and K. Tuchin, Phys. Lett., B663, 217 (2008).

[21] C. Sasaki, and K. Redlich, arXiv: 0806.4745 [hep-ph]; 0811.4708 [hep-ph].

[22] S.S. Gubster, S.S. Pufu, and F.D. Rocha, arXiv:0806.0407 [hep-th].

[23] S. Sakai, and A. Nakamura, PoS LAT2007:221 (2007).

[24] J. Berges, and K. Rajagopal, Nucl. Phys. B538, 215 (1999).

[25] M.A. Stephanov, K. Rajagopal, and E.V. Shuryak, Phys. Rev. Lett., 81, 4816 (1998).

[26] C.R. Allton et al., Phys. Rev. D71, 054508 (2005); R.V. Gavai, and S. Gupta, Phys. Rev. D71, 114014 (2005)

[27] Z. Fodor, and S.D. Katz, JHEP 0404, 050 (2004); F. Csikor, G. I. Egri, Z. Fodor, S. D. Katz, K. K. Szabo and A. I. Toth, JHEP 0405, 046 (2004)

[28] V. Koch, A. Majumder, and J. Randrup, Phys. Rev. C72, 064903 (2005).

[29] B. Berdnikov, and K. Rajagopal, Phys. Rev. D61, 105017 (2000).

[30] C. Sasaki, B. Friman, and K. Redlich, Phys. Rev. Lett. 99, 232301 (2007); Phys. Rev. D77, 034024 ( 2008); Mod. Phys. Lett., A23, 2469 (2008).

[31] . O. Scavenius, A. Dumitru, and A.D. Jackson, Phys. Rev. Lett. 87, 182302 (2001); G. Torrieri, B. Tomasik, and I. Mishustin, Phys. Rev. C77, 034903 (2008).

[32] G. Röpke, L. Münchow, and H. Schulz, Phys. Lett. B110, 21 (1982).

[33] H. Schulz, D.N. Voskresensky, and J. Bondorf, Phys. Lett. B133, 141 (1983). 
[34] A.D. Panagiotou, M.W. Curtin, H. Toki, D.K. Scott, and P.J. Siemens, Phys. Rev. Lett. 52, 496 (1984).

[35] M. D’Agostino et all., Phys. Lett. B473, 219 (2000); M. Schmidt et all., Phys. Rev. Lett. 86, 1191 (2001).

[36] P. Chomaz, M. Colonna, and J. Randrup, Phys. Rep. 389, 263 (2004).

[37] N.K. Glendenning, Phys. Rev. D46, 1274 (1992); Phys. Rep. 342, 393 (2001).

[38] H. Heiselberg, C.J. Pethick, and E.F. Staubo, Phys. Rev. Lett. 701355 (1993); D.N. Voskresensky, M. Yasuhira, and T. Tatsumi, Nucl.Phys. A723, 291 (2003); Toshiki Maruyama, T. Tatsumi, D.N. Voskresensky, T. Tanigawa, and S. Chiba, Phys. Rev. C72, 015802 (2005); Toshiki Maruyama, T. Tatsumi, D.N. Voskresensky, T. Tanigawa, T. Endo, and S. Chiba, Phys. Rev. C73, 035802 (2006).

[39] G. Watanabe, Phys. Rev. A73, 013616 (2006).

[40] V.V. Skokov, and D.N. Voskresensky, arXiv: 0811.3868 [nucl-th].

[41] J. W. Cahn, and J. E. Hilliard, J. Chem. Phys 28, 258 (1958).

[42] D. Bower, and S. Gavin, Phys. Rev. C64, 051902 (2001);

[43] L.P. Csernai, and J. Kapusta, Phys. Rev. D46, 1379 (1992).

[44] T. Koide, G. Krein, and R. O. Ramos, Phys. Lett. B636, 96 (2006).

[45] S. Coleman, Phys. Rev. D15, 2929 (1977); E.J. Copeland, M. Gleiser, and H.R. Müller, Phys. Rev. D52, 1920 (1995).

[46] D.A. Beysens, and Y. Garrabos, Physica A281, 361 (2000).

[47] K. Morawetz, M. Ploszajczak, and V.D. Toneev, Phys. Rev. C62, 064602 (2000).

[48] A.S. Khvorostukhin, V.D. Toneev, and D.N. Voskresensky, Nucl. Phys. A791, 180 (2007); Nucl. Phys. A813, 313 (2008).

[49] Y. Aoki, Z. Fodor, S. D. Katz, and K. K. Szabo, JHEP 0601, 089 (2006).

[50] M. S. Berger, and R. L. Jaffe, Phys. Rev. C 35, 213 (1987) ; C44, R566 (1991).

[51] J. Randrup, arXiv:0903.4736; Phys. Rev. C79, 054911 (2009). 